\title{
Epac2 Elevation Reverses Inhibition by Chondroitin Sulfate Proteoglycans In Vitro and Transforms Postlesion Inhibitory Environment to Promote Axonal Outgrowth in an Ex Vivo Model of Spinal Cord Injury
}

\author{
DDAlba Guijarro-Belmar, ${ }^{1}$ Mindaugas Viskontas, ${ }^{1}$ Yuting Wei, ${ }^{1}$ Xuenong Bo, ${ }^{2}$ Derryck Shewan, ${ }^{1}$ and Wenlong Huang ${ }^{1}$ \\ ${ }^{1}$ Institute of Medical Sciences, School of Medicine, Medical Sciences \& Nutrition, University of Aberdeen, Aberdeen AB25 2ZD, United Kingdom, and \\ ${ }^{2}$ Center for Neuroscience, Surgery and Trauma, Queen Mary University of London, London E1 2AT, United Kingdom
}

Millions of patients suffer from debilitating spinal cord injury (SCI) without effective treatments. Elevating cAMP promotes CNS neuron growth in the presence of growth-inhibiting molecules. cAMP's effects on neuron growth are partly mediated by Epac, comprising Epac1 and Epac2; the latter predominantly expresses in postnatal neural tissue. Here, we hypothesized that Epac2 activation would enhance axonal outgrowth after SCI. Using in vitro assays, we demonstrated, for the first time, that Epac2 activation using a specific soluble agonist (S-220) significantly enhanced neurite outgrowth of postnatal rat cortical neurons and markedly overcame the inhibition by chondroitin sulfate proteoglycans and mature astrocytes on neuron growth. We further investigated the novel potential of Epac2 activation in promoting axonal outgrowth by an ex vivo rat model of SCI mimicking post-SCI environment in vivo and by delivering S-220 via a self-assembling Fmoc-based hydrogel that has suitable properties for SCI repair. We demonstrated that S-220 significantly enhanced axonal outgrowth across the lesion gaps in the organotypic spinal cord slices, compared with controls. Furthermore, we elucidated, for the first time, that Epac2 activation profoundly modulated the lesion environment by reducing astrocyte/microglial activation and transforming astrocytes into elongated morphology that guided outgrowing axons. Finally, we showed that S-220, when delivered by the gel at 3 weeks after contusion SCI in male adult rats, resulted in significantly better locomotor performance for up to 4 weeks after treatment. Our data demonstrate a promising therapeutic potential of S-220 in SCI, via beneficial effects on neurons and glia after injury to facilitate axonal outgrowth.

Key words: astrocyte; axonal regrowth; cAMP; Epac2; organotypic; spinal cord injury

Significance Statement

During development, neuronal cAMP levels decrease significantly compared with the embryonic stage when the nervous system is established. This has important consequences following spinal cord injury, as neurons fail to regrow. Elevating cAMP levels encourages injured CNS neurons to sprout and extend neurites. We have demonstrated that activating its downstream effector, Epac2, enhances neurite outgrowth in vitro, even in the presence of an inhibitory environment. Using a novel biomaterial-based drug delivery system in the form of a hydrogel to achieve local delivery of an Epac2 agonist, we further demonstrated that specific activation of Epac2 enhances axonal outgrowth and minimizes glial activation in an ex vivo model of spinal cord injury, suggesting a new strategy for spinal cord repair.

\section{Introduction}

No cure for spinal cord injury (SCI) exists due to the complex injury nature. Obstacles to SCI repair include a lack of intrinsic

Received Feb. 15, 2019; revised Aug. 5, 2019; accepted Aug. 8, 2019.

Author contributions: A.G.-B., X.B., D.S., and W.H. designed research; A.G.-B., M.V., Y.W., D.S., and W.H. performed research; A.G.-B., M.V.,Y.W., X.B., D.S., and W.H. analyzed data; A.G.-B. and W.H. wrote the first draft of the paper; A.G.-B., M.V., X.B., D.S., and W.H. edited the paper; A.G.-B., D.S., and W.H. wrote the paper; X.B. contributed unpublished reagents/analytic tools.

This work was supported by the International Spinal Research Trust, Scottish Rugby Union, and RS McDonald Charitable Trust. We thank Professor Divya Chari for training with the ex vivo model; Dr. Amer Syed, Elena Moratal- growth capacity of adult mammalian CNS neurons, cavity and glial scar formation, and inhibitory molecules expressed at SCI lesions including chondroitin sulfate proteoglycans (CSPGs) and Nogo (Fawcett, 2006; Cregg et al., 2014). Multiple strategies have

Torres, James W. Thomson, Victoria Torsteinsbø, and Dr. Marieta Georgieva for assistance with rheology and in vitro experiments.

The authors declare no competing financial interests.

Correspondence should be addressed to Wenlong Huang at w.huang@abdn.ac.uk.

https://doi.org/10.1523/JNEUROSCI.0374-19.2019

Copyright $\odot 2019$ the authors 
been developed to boost the intrinsic growth capacity of adult CNS neurons but without translational success. For example, neurotrophins enhance axonal regrowth into the lesion in rodent SCI models, but their inability to overcome inhibitory molecules makes them largely ineffective as a treatment (Hannila and Filbin, 2008). In adult mice, genetic Klf7 overexpression enhances corticospinal tract axonal regrowth into the lesion with pyramidotomy (Blackmore et al., 2012) but fails to promote regrowth beyond the lesion after SCI (Wang et al., 2017). Genetic PTEN deletion promotes axonal regrowth beyond the lesion in rodent SCI models (Danilov and Steward, 2015; Du et al., 2015; Zhou et al., 2015), but potential oncogenesis and long-term harmful effects on neurons pose concerns (Gutilla and Steward, 2016; Gutilla et al., 2016).

Elevating cAMP levels has been shown as one of the most effective ways to promote axonal regeneration and functional recovery in preclinical SCI research (Neumann et al., 2002; Qiu et al., 2002; Nikulina et al., 2004; Costa et al., 2013). Moreover, cAMP elevation overcomes inhibition on axonal outgrowth caused by myelin-associated inhibitors (Siddiq and Hannila, 2015). However, targeting cAMP is unlikely to be translated to the clinic as it is ubiquitously expressed, so its manipulation will alter functions in all types of cells. Therefore, a downstream target of cAMP more localized to neuronal cells may offer an alternative solution.

Traditionally, it was thought that cAMP solely signals through protein kinase $\mathrm{A}$ (PKA) to manifest its effects on axonal regeneration. However, the development of more specific tools allowed the identification of Epac, a guanine nucleotide exchange factor for Rap1, as an intracellular target, other than PKA, directly activated by cAMP (Cheng et al., 2008). Indeed, a recent study showed that the cortical infusion of a PKA antagonist, contrary to the traditional hypothesis, led to a significant increase in functional recovery in rats with SCI, suggesting the involvement of a different cAMP downstream effector in promoting axon regeneration (Wei et al., 2016).

In our previous studies, knocking down intracellular Epac in adult rat DRG neurons using siRNA led to a significant reduction in neurite outgrowth that could not be rescued by the addition of cAMP analogs (Murray and Shewan, 2008). In addition, chemoattraction assays showed that axons are similarly attracted to a gradient of an Epac agonist as they are to a gradient of a cAMP agonist (Murray et al., 2009). Furthermore, the addition of Epac agonists to an in vitro SCI remyelination model significantly increased myelination and neurite outgrowth compared with controls (Boomkamp et al., 2014). Together, these studies suggest that Epac could be the key protein mediating the positive effects of cAMP on axonal growth and guidance in vitro (Murray and Shewan, 2008; Murray et al., 2009; Peace and Shewan, 2011). Epac has two isoforms: Epac1 is widely expressed embryonically, whereas Epac2 is restricted mainly to postnatal nervous tissue (Peace and Shewan, 2011), suggesting that targeting Epac2 could provide a neuronspecific route for manipulation to enhance axonal growth.

Therefore, our hypothesis was that the elevation of Epac2 activity by a specific agonist would enhance neurite outgrowth in vitro and promote axonal outgrowth in an ex vivo model that mimics the in vivo inhibitory environment after SCI. To achieve a gradual, sustained, and local release of the Epac2 agonist in the injury site, we explored the use of a novel self-assembling Fmocbased hydrogel as a depot that can be directly injected into the injury site, thus representing a minimally invasive surgical procedure for future clinical translation (Zhu and Marchant, 2011; Tukmachev et al., 2016).

\section{Materials and Methods}

All procedures involving the use of live animals and animal tissues were performed in accordance with the UK Home Office (Scientific Procedures) Act, 1986, and were approved by the local ethics committee of the University of Aberdeen.

Cortical neuron culture. Cortices of Sprague Dawley rats at postnatal days $0-1$ (mixed sexes) were harvested as a source for culturing cortical neurons. The tissue was dissociated enzymatically with $50 \mathrm{U} / \mathrm{ml}$ papain (Worthington) in retinal buffer at $\mathrm{pH} 7.4$ composed of 15 mM HEPES (Sigma-Aldrich) buffered Hanks balanced salt solution (Invitrogen) containing $300 \mu \mathrm{M}$ D-L cysteine (Sigma-Aldrich) and incubated at $37^{\circ} \mathrm{C}$ for $30 \mathrm{~min}$. The papain action was stopped by using 10\% FBS (Thermo Fisher Scientific), and cells were resuspended in Neurobasal medium (Thermo Fisher Scientific) supplemented with 2\% B-27 (Thermo Fisher Scientific), 1\% Glutamax (Thermo Fisher Scientific), and $100 \mathrm{U} / \mathrm{ml}$ penicillin and $100 \mu \mathrm{g} / \mathrm{ml}$ streptomycin (Sigma-Aldrich). Cortical neurons were plated at 40,000 neurons $/ \mathrm{ml}$ on round $13 \mathrm{~mm}$ glass coverslips (BDH) coated overnight with $10 \mu \mathrm{g} / \mathrm{ml}$ poly-D-lysine (PDL; SigmaAldrich) and cultured for up to $48 \mathrm{~h}$ at $37^{\circ} \mathrm{C}$ in a $5 \% \mathrm{CO}_{2} / 95 \%$ air incubator (NU-581DE; Nuaire).

$D R G$ neuron and explant cultures. DRGs were dissected from Sprague Dawley rats at postnatal days 0-5 (mixed sexes), collected in Ham's F12 medium (Thermo Fisher Scientific), and trimmed to remove roots. Explants were plated directly when needed. For dissociating DRG neurons, ganglia were transferred to $1 \mathrm{ml}$ retinal buffer containing $50 \mathrm{U} / \mathrm{ml}$ papain as described above. The tissue was then transferred to $100 \mu \mathrm{l} \mathrm{Hanks}$ balanced salt solution containing $0.25 \mathrm{mg} / \mathrm{ml}$ trypsin inhibitor (SigmaAldrich) and $50 \mu \mathrm{g} / \mathrm{ml}$ DNase (Sigma-Aldrich), followed by trituration using a Gilson P200 pipette until a single-cell suspension was achieved. The dissociated neurons were diluted to the required density with Neurobasal medium supplemented as described above plus nerve growth factor (100 ng/ml; Sigma-Aldrich). DRG neurons were plated at 5000 neurons/ml on $13 \mathrm{~mm}$ coverslips coated overnight with PDL as described above and $2 \mu \mathrm{g} / \mathrm{ml}$ laminin (Thermo Fisher Scientific) and cultured for up to $48 \mathrm{~h}$ at $37^{\circ} \mathrm{C}$ in a $5 \% \mathrm{CO}_{2} / 95 \%$ air incubator.

Microglial and astrocyte cultures. Primary mixed microglia and astrocytes were cultured as previously described (Georgieva et al., 2018) from the cortices of Sprague Dawley rats at postnatal days 3-6 (mixed sexes) and plated on $10 \mu \mathrm{g} / \mathrm{ml}$ PDL (Sigma-Aldrich) coated $60 \mathrm{~mm}$ Petri dishes (Biofil Triple red TCD010060). After $4 \mathrm{~d}$ of incubation at $37^{\circ} \mathrm{C}$ in a $5 \%$ $\mathrm{CO}_{2} / 95 \%$ air incubator, the presence of two layers of cells was confirmed: one layer of astrocytes firmly attached to the surface of the dish and a layer of microglia visible as large spherical bright cells on top of the astrocyte layer. For microglia plating, medium was collected after a gentle swirl, centrifuged at $200 \times g$ for $10 \mathrm{~min}$ at $4^{\circ} \mathrm{C}$ and the pellet resuspended in warm medium. The microglia suspension was plated on $18 \mathrm{~mm}$ uncoated sterile glass coverslips and used the following day. For astrocyte plating, once microglial cells were collected, cultures were washed repeatedly with warm PBS and incubated with trypsin/EDTA 0.25\% (SigmaAldrich) for $5 \mathrm{~min}$ at $37^{\circ} \mathrm{C}$. Trypsin action was stopped by addition of $10 \% \mathrm{FBS}$, centrifuged at $200 \times \mathrm{g}$ for $10 \mathrm{~min}$ at $4^{\circ} \mathrm{C}$, and replated in two PDL-coated flasks. Cultures were maintained for 3 weeks to ensure maturation of the astrocytes (Smith et al., 1990). Medium was changed every $2 \mathrm{~d}$, and cells were split when confluent. Microglia and astrocytes were maintained in medium composed of DMEM with 10\% FBS and 100 $\mathrm{U} / \mathrm{ml}$ penicillin and $100 \mu \mathrm{g} / \mathrm{ml}$ streptomycin.

Soluble agonist and antagonist treatments for neurons in vitro. Soluble agonist Sp-8-BnT-cAMPS (S-220) and antagonist ESI-05 (both Biolog) were used according to the manufacturer's instructions for specific Epac2 activation and inactivation in neurons, respectively. In some experiments, chicken extracellular CSPGs (CC117; Sigma-Aldrich) were added to culture media at $0.5 \mu \mathrm{g} / \mathrm{ml}$ at the time of cell plating.

In vitro treatments for microglia and astrocytes. Mature astrocytes were plated in $13 \mathrm{~mm}$ PDL-coated coverslips at a density of 45,000 cells/ml. Microglia were plated in $18 \mathrm{~mm}$ uncoated coverslip at a density of 70,000 cells $/ \mathrm{ml}$. Both cell types were allowed to attach and grow overnight. The next morning, media were changed to serum-free media before treatments. Three conditions were studied: control, lipopolysaccharide 
(LPS), and simultaneous LPS and S-220 treatment. Therefore, $10 \mu \mathrm{g} / \mathrm{ml}$ LPS was added to the media alone or together with S-220 at $2.5 \mu \mathrm{M}$, a concentration showing effects in promoting neurite outgrowth. The treatments were left for $4 \mathrm{~h}$.

siRNA transfection. For siRNA knockdown, DRG neurons were transfected with $3 \mu \mathrm{g}$ of siRNA duplex (Epac2: ATC CGT GAA TGT AGT CAT TTA) (QIAGEN) using the Amaxa Nucleofector II device (Lonza Walkersville) according to the manufacturer's instructions. To establish transfection efficiency using this method, $3 \mu \mathrm{g}$ of $3^{\prime}$-fluorescein-tagged Allstars negative control siRNA (QIAGEN) was transfected into neonatal DRG neurons, which were plated on $2 \mu \mathrm{g} / \mathrm{ml}$ laminin-coated glass coverslips. After $16 \mathrm{~h}$, cells were fixed in 4\% PFA (Sigma-Aldrich), immunolabeled using anti-GAP43, and analyzed using an AF6000LX microscope (Leica Microsystems). Fluorescein-labeled neurons were compared with the total number of GAP43-positive neurons to obtain the transfection efficiency, which was calculated as $80 \%$.

Lentiviral vector $(L V)$ production. We had technical limitations for the construction of LVs carrying the specific Epac2 cDNA because the available sequence was not suitable for the subcloning in the lentiviral transfer vector. Therefore, as a proof of concept, we constructed Epac-LV as follows. Human Epac1-YFP construct was kindly provided by Dr. Kees Jalink at The Netherlands Cancer Institute (Amsterdam, The Netherlands). hEpac1-YFP cDNA was subcloned downstream of the CMV promoter in the lentiviral transfer vector pRRL. Self-inactivating LVs were made by cotransfecting HEK293T cells using a three-plasmid system (Dull et al., 1998). pRRL/hEpac1-YFP or pRRL/GFP plasmids were transfected together with pPACK and pENV plasmids using the calcium precipitation method (Dull et al., 1998). Lentiviral particles were harvested $45 \mathrm{~h}$ after transfection and concentrated using polyethylene glycol precipitation. Viral particles were resuspended in sterile PBS after precipitation. The titers were determined by transduction of HEK293T cells using serially diluted viral stocks. The titers were $4.6 \times 10^{9} \mathrm{TU} / \mathrm{ml}$ for LV/hEpacl-YFP (referred as LV/Epac hereafter), and $2.2 \times 10^{9} \mathrm{TU} / \mathrm{ml}$ for LV/GFP. The viral stocks were aliquoted and stored at $-80^{\circ} \mathrm{C}$. Cortical neurons to be transduced with the LV were plated in 24 well plates (Greiner) at a density of 60,000 cells $/ \mathrm{ml} ; 2.2 \times 10^{6} \mathrm{TU}$ of LV/GFP or LV/hEpac1-YFP was added per well to allow transduction. Media were replaced the day after and then changed every $2 \mathrm{~d}$. Cells were maintained for $7 \mathrm{~d}$ to ensure full expression of the transgenes. Anti-GFP-labeled neurons were compared with the total number of $\beta$-tubulin-(III) -positive neurons to obtain the transduction efficiency.

FRET SE microscopy. Fluorescent resonance energy transfer sensitized emission (FRET SE) was performed in live DRG neurons as previously described (Tucker, 2014). FRET SE microscopy allowed us to demonstrate the level of Epac2 protein activity in the presence of the agonist, S-220, or the antagonist, ESI-05, respectively, in the growth cones of live DRG neurons. Two fluorophores (CFP and YFP) were fused to the two termini of Epac2 protein in the Epac FRET construct (see below). When the distance between the two is $<10 \mathrm{~nm}$, which occurs when the protein is inactive, a FRET signal is produced. On activation of the protein, a conformational change occurs and the distance between the two fluorophores becomes $>10 \mathrm{~nm}$, abolishing the FRET signal. Thus, high FRET signals correlate with low Epac2 activation and low FRET signals correlate with high Epac2 activation. Briefly, neurons were transfected with the construct mTurqDel-Epac (dDEPCD) cp173Venus (d)-Venus (d) (H74) (Klarenbeek et al., 2011) (kindly provided by Dr. Kees Jalink, The Netherlands Cancer Institute, Amsterdam, The Netherlands) using the Amaxa Nucleofector II device (Lonza Walkersville). The cells were diluted in medium after transfection and cultured in $50 \mathrm{~mm}$ glassbottomed dishes (MatTek) coated with PDL and $5 \mu \mathrm{g} / \mathrm{ml}$ laminin. One day after transfection, the medium was topped up to $5 \mathrm{ml}$ and buffered with 15 mM HEPES buffer. Using an AF6000LX imaging system (Leica Microsystems) configured correctly for FRET, images were taken of a selected growth cone in the CFP, YFP, and FRET channels every 2 min for a total of $30 \mathrm{~min}$. For each experiment, the first four images were taken without application to act as a baseline as the system stabilized; then the reagent was added after the fourth time point. The Epac2 agonist (S-220) was diluted to a concentration of $5 \mu \mathrm{M}$ and the antagonist (ESI-05) to 10 $\mu \mathrm{M}$. Analysis of each image (combining all 3 channels) was performed using Application Suite-Advanced Fluorescence software (Leica Microsystems) by selecting a "region of interest" within the growth cone and recording the FRET value. These data were then expressed as a percentage change from time 0 .

Choice assay. Mature astrocytes obtained as described above were plated at 5000 cells $/ \mathrm{ml}$ on $5 \mu \mathrm{g} / \mathrm{ml}$ laminin custom-made dishes. These dishes consisted of $60 \mathrm{~mm}$ Petri dishes (CellStar, Greiner Bio-One) that had three 13-mm-diameter holes in the base of the dish using a hole puncher; $18 \mathrm{~mm}$ coverslips were mounted onto the base of the dish using RTV 3140 coating glue (Dow Corning) that is nontoxic to cells. Astrocytes were left to grow for $5 \mathrm{~h}$ before plating of DRG neurons among the astrocytes. Cocultures were further incubated at $37^{\circ} \mathrm{C}$ overnight in a $\mathrm{CO}_{2}$ incubator. S-220 was added just before time lapse imaging. Time lapse recordings were taken over 30-60 min using NIS-element software and an Okolab chamber-mounted eclipse Ti inverted microscope (Nikon). The response of each DRG neurite was categorized into two different categories: crossover and reflect/retract. Each response was calculated as a percentage of the total number of interactions in each experimental condition, control or S-220 treatment. Data were collated for at least 10 growth cones per condition and pooled from at least 3 separate experiments.

Growth cone turning assays. Assays were performed as previously described (Murray and Shewan, 2008). Briefly, DRG neurons were cultured in low-walled glass-bottomed $50 \mathrm{~mm}$ Petri dishes (MatTek) coated with $5 \mu \mathrm{g} / \mathrm{ml}$ laminin. The culture medium, supplemented with $15 \mathrm{~mm}$ HEPES buffer, was overlaid with a thin monolayer of warmed vegetable oil and transferred to a Nikon Diaphot inverted-stage microscope equipped with a stage-mounted incubator heated to $37^{\circ} \mathrm{C}$. Growth cones were visualized using a Nikon Diaphot inverted microscope connected to a PC running QWin version 2.1 software (Leica Microsystems). Borosilicate glass micropipettes (Intracel) were pulled in a Sutter pipette puller (Intracel) so that they had a bore width of $1 \mu \mathrm{m}$. Growth cones were oriented at $45^{\circ}$ and $100 \mu \mathrm{m}$ from a glass micropipette containing 3-5 $\mu \mathrm{l}$ of either F12 as a control or S-220 treatment. Each reagent was ejected by an air pulse of 3 psi at $2 \mathrm{~Hz}$ and $10 \mathrm{~ms}$ duration, applied to the pipette by a Picospritzer III (Intracell). Each growth cone was assayed for $30 \mathrm{~min}$, and only growth cones that had advanced at least $10 \mu \mathrm{m}$ from the original position at time 0 were included. The angle between the position of the growth cone at $30 \mathrm{~min}$, its original position at time 0 , and its original direction of growth was calculated using ImageJ software. Data were collated for at least 10 growth cones per condition, pooled from at least 3 separate experiments.

Hydrogel characterization. Fmoc hydrogel is commercially available (BiogelX) and can also be made using published methods (Alakpa et al., 2016). Hydrogel was prepared at $2.2 \mathrm{mg} / \mathrm{ml}$ using ThinCert cell inserts (Greiner Bio-One) and following the manufacturer's instructions. RGD (Arg-Gly-Asp) peptide (ab142698; Abcam) at different concentrations was incorporated into the gel according to the manufacturer's instructions. Rheology experiments (Discovery HR-2, TA Instruments) were performed at different time points during degradation to assess changes in gel stiffness, using Frequency sweep test $0-100 \mathrm{~Hz}, 1 \%$ strain at $37^{\circ} \mathrm{C}$. Gels were also weighed at different time points during degradation in PBS. Buffer was completely aspirated, and the insert weight was subtracted from the total weight. The Griess assay was used to quantify nitrite release by primary microglia when cultured with the hydrogel for $6 \mathrm{~h}$. LPS ( $1 \mu \mathrm{g} / \mathrm{ml}$; Sigma-Aldrich) was used as a positive activated control. Fluorescein sodium salt (Sigma-Aldrich) was used to assess the release profile since it has similar molecular weight compared with S-220 $(\mathrm{MW}=376.27 \mathrm{Da})$ and can be measured by a fluorescence plate reader (Omega microplate reader; BMG Labtech) at 440-520 nm with $700 \mathrm{~ms}$ gain (Perale et al., 2012; Wilems and Sakiyama-Elbert, 2015).

Immunocytochemistry. Cells and explants were fixed with 4\% PFA in PBS for $30 \mathrm{~min}$, followed by incubation with $10 \%$ normal goat serum for $1 \mathrm{~h}$, all at room temperature. Cells/explants were then incubated with primary antibodies overnight at $4^{\circ} \mathrm{C}$. We used the following primary antibodies: anti- $\beta$-tubulin-III (1:1000, mouse; RRID:AB_1844090; Sigma-Aldrich), anti-growth associated protein-43 (GAP-43) (1:500, rabbit; RRID:AB_10622060; Sigma-Aldrich), anti-glial fibrillary acidic protein (GFAP) (1:1000, mouse; RRID:AB_94844; Merck Millipore), 
anti-ionized calcium binding adaptor molecule-1 (Iba-1) (1:1000; rabbit; RRID:AB_2314666; Wako), anti-inducible nitric oxide synthase (iNOS) (1:100; mouse; RRID:AB_397719; BD Biosciences), and anti-GFP (1: 100, chicken; RRID:AB_300798; Abcam). Following three washes with PBS, cells/explants were incubated for $2 \mathrm{~h}$ at room temperature with the appropriate secondary antibodies, including goat anti-mouse, anti-rabbit, or anti-chicken antibodies (1:400, RRID:AB_221544, AB_221544, AB_143160, AB_141672, and AB_141359, respectively; Invitrogen). Coverslips were mounted with PBS/glycerol (1:8 ratio) after counterstaining with Hoechst $33342(2 \mu \mathrm{g} / \mathrm{ml}$ in PBS; Sigma-Aldrich). All primary/secondary antibodies and goat serum were prepared with PBS containing $0.2 \%$ Triton X-100 (Sigma-Aldrich) and $0.1 \%$ sodium azide (Sigma-Aldrich).

Imaging and quantification for neurite outgrowth. An Eclipse Ti-E microscope (Nikon) was used to acquire all images. HCA-vision (CSiRO) was used to quantify total neurite length. The plugin Neuron J for ImageJ was used to manually quantify Maximal Neurite Length of dissociated neurons and maximal distance $\left(\mathrm{D}_{\max }\right)$ of neurite growth from DRG explants (Torres-Espín et al., 2014). Analyses were performed for at least 100 neurons and 10 DRG explants per condition from three different experiments.

Analysis of astrocytes and microglia activation in vitro. For each coverslip, 10 representative micrographs were taken with a $20 \times$ objective. A minimum of 100 cells per condition were analyzed. The activation of the astrocytes was determined by the quantification of GFAP intensity (Sofroniew, 2009). All images were converted into 8-bit files, and the function threshold in ImageJ was applied to select only area with GFAP immunoreactivity. Intensity of staining was determined by the mean optical density value. The mean background fluorescence proximate to each cell was subtracted from the measured fluorescence intensity of the cell area to give a corrected fluorescence intensity. The activation of microglia was quantified by calculating the percentage of iNOS/Iba-1 double-immunoreactive cells in the total Iba-1-immunoreactive cells. Data for same conditions from three different replicates were averaged and compared.

Production of spinal cord slices: ex vivo modeling of SCI. The ex vivo model using rat spinal cord tissue was adapted from a study using mouse spinal cord tissue (Weightman et al., 2014). The SD pup's (postnatal days $0-3$; mixed sexes) dorsal trunk skin surface was sprayed with $95 \%$ ethanol; then a dorsal midline incision was made and skin flaps were retracted to expose the spinal column. A midline incision was made along the length of the spine using fine micro-dissecting Vannas spring scissors (Fine Science Tools). The spinal cord was rapidly dissected from the thoracolumbar region and placed in ice-cold slicing media. The meninges were gently removed, and a $0.7-0.9 \mathrm{~cm}$ length of cord was cut from the thoracic region. The cord was transferred onto an ice-cold chopping plate of a preset McIlwain tissue chopper and sliced lengthways in the parasagittal plane (350 $\mu \mathrm{m}$ thickness). Three or four slices could be derived from each cord. Slices were incubated in ice for $90 \mathrm{~min}$ and then selected under a dissecting microscope and transferred to precut Omnipore membrane "confetti" (Merck Millipore), resting on the Mill cell culture insert membrane (Merck Millipore), using a plastic Pasteur pipette. The process of slice plating was performed under a dissecting microscope to ensure the correct position of the slice. To avoid variability, the selected slices from each pup were plated together in one dish fitting up to four slices. Slices were cultured at the air-medium interface for up to $10 \mathrm{~d}$ in a humidified incubator with $5 \% \mathrm{CO}_{2}$ at $37^{\circ} \mathrm{C}$ with $80 \%$ medium changes every other day. The experimental unit, $n$, refers to slice numbers obtained from different pups.

Lesioning organotypic spinal cord slice cultures. Cords were lesioned after $3 \mathrm{~d}$ in culture. A slice lesioning tool was custom-made using a preassembled, double-bladed scalpel with a spacer creating a gap of $\sim 700$ $\mu \mathrm{m}$. The tool was aseptically assembled before lesioning by taping together two surgical blades (size 15) with a spacer in the middle secured into a scalpel holder. The same lesioning tool was used in each individual experiment. The lesioning procedure was implemented inside a horizontal laminar flow hood under a dissecting microscope. To stabilize the slice in the process of lesioning, the confetti was cut in a $\mathrm{T}$ shape, allowing it to be secured with forceps while producing the lesion. A small lateral move- ment was used to ensure complete transection of the cord without damaging the confetti. An aspirator fitted with a pipette tip was used to remove all the debris resulting from the lesioning.

Assessment of the viability of spinal cord slices. To assess viability, three intact slices were randomly chosen before and after the lesion and incubated with the live/dead assay kit (Invitrogen) containing calcein $(1 \mu \mathrm{l} /$ $\mathrm{ml}$ ) for live cells and ethidium bromide $(3 \mu \mathrm{l} / \mathrm{ml})$ for dead cells, for 15 $\min$ at $37^{\circ} \mathrm{C}$. Following the incubation period, slices were mounted with antifade mounting media Vectashield (Vector Laboratories). Immediately afterward, fluorescence micrographs were taken $(664 \mu \mathrm{m} \times 834$ $\mu \mathrm{m})$ with a $10 \times$ objective with consistent exposure settings and converted to grayscale. The integrated density (mean gray value per unit area) was measured from both live- and dead-stained micrographs. Thus, the viability for each slice was calculated by expressing the corrected integrated density of the live-stained micrograph as a percentage of the sum total from both stained groups (Cho et al., 2009). The quantification was conducted using ImageJ software.

Treatment of spinal cord slice. Treatment was administered immediately after the lesion either in the medium or incorporated into the gels. Treatment gels were prepared $2 \mathrm{~h}$ before lesioning and precut into 700 $\mu \mathrm{m} \times 700 \mu \mathrm{m}$ pieces using the Mcllwain tissue chopper. Gels were incorporated into the injury site under the microscope using needles. Treatment was applied for $7 \mathrm{~d}$.

Immunohistochemistry. All slices were fixed with $4 \%$ PFA $7 \mathrm{~d}$ after the lesion for $30 \mathrm{~min}$, followed by incubation with $10 \%$ normal goat serum for $1 \mathrm{~h}$ at room temperature. Slices were then incubated with primary antibodies for $24 \mathrm{~h}$ at $4^{\circ} \mathrm{C}$. Primary antibodies used were as follows: anti- $\beta$-tubulin-III (1:1000, mouse), anti-GFAP (1:500, rabbit; RRID: AB_2109645; Merck Millipore), anti-GFAP (1:1000, mouse), antiNestin (1:400, mouse; RRID:AB_2151130; Merck Millipore), and antiIba-1 (1:1000; rabbit). Following three washes with PBS, cells were incubated for $4 \mathrm{~h}$ at room temperature with appropriate secondary antibodies, including goat anti-mouse or anti-rabbit secondary antibodies. Slices were mounted with Vectashield. All primary/secondary antibodies and goat serum were prepared with PBS containing $0.2 \%$ Triton X-100 and $0.1 \%$ sodium azide.

Image and axonal outgrowth analysis on spinal cord slices. All fluorescence images were captured with an Eclipse Ti-E microscope (Nikon). Single panoramic images were generated when appropriate using the Large Image tool in Nis Elements for advanced research software. Maximal Intensity Projection images using multiple $Z$ planes were also used when appropriate. Where applicable, fluorescence images of immunostained slices were merged using Photoshop. Quantitative analysis of $\beta$-tubulin-III stained profiles was conducted across the lesion gap from one lesion margin to the other according to previously published methods (Weightman et al., 2014), with 20 division lines at an equal distance of $35 \mu \mathrm{m}$ between each line using a multiselection plugin from ImageJ. Peak analysis plugin from ImageJ was then used to convert the staining into optical density at each division line covering the whole width of the lesion gap, which is equal to the width of the slice. Finally, the peaks (number of staining profiles) per square millimeter per slice (Weightman et al., 2014) were calculated by averaging the optical densities and then divided by the area of the lesion gap.

Analysis of astrocyte activation in spinal cord slices. Quantitative analysis of astrocyte activation was conducted by semiquantification of GFAP staining intensity within the lesion gap. Thus, three fluorescence images $(478 \mu \mathrm{m} \times 598 \mu \mathrm{m})$ were taken with a $20 \times$ objective from the front areas of the GFAP-immunoreactive glial scar from one-half of the lesion first: the central one aligned with the midline perpendicular to the lesion margin and then one right and one left with equal distance of $200 \mu \mathrm{m}$ from the central one. We then repeated with another three fluorescence images for the other half of the lesion as described above. Consistent exposures were applied for all captured images, which were then converted to grayscale followed by applying a threshold using the ROI manager plugin from ImageJ. Finally, the intensity of the GFAP staining from the six images per slice was averaged.

Reactivity of astrocytes was also estimated by the overlapping of GFAP and Nestin as previously described (O'Neill et al., 2017). Thus, three fluorescence images $(478 \mu \mathrm{m} \times 598 \mu \mathrm{m})$ were taken with a $20 \times$ objec- 
tive from the front areas of the GFAP-Nestinimmunoreactive glial scar from both halves of the lesion as described above. Briefly, captured images were converted to grayscale followed by the application of the "auto-threshold" function using the "Default" setting in ImageJ. This generates a binary image with a black signal in a white background. "Image Calculator" function in ImageJ using the "AND" operation was used to generate a third image representing the regions of overlap between binary green and red. The number of overlapping pixels was calculated by using the "Histogram" function in ImageJ to obtain the total number of pixels with value 255 (black). A minimum of three images was analyzed per spinal cord slice and averaged to get a final value per slice.

Analysis of microglial activation in spinal cord slices. Quantitative analysis of microglial activation was conducted by semiquantification of the cell body perimeter of Iba-1-immunoreactive cells within the lesion gap. Thus, three fluorescence images $(316 \mu \mathrm{m} \times 395 \mu \mathrm{m})$ were taken with a $30 \times$ objective as described above for astrocyte activation analysis. The perimeter (Kozlowski and Weimer, 2012) of at least 20 Iba-1-immunoreactive cells per micrograph was then measured using ImageJ and averaged for each slide. For nonlesioned microglial activation analysis, three images were taken from at least $1 \mathrm{~mm}$ away from the lesion site, where Iba-1-immunoreactive cells displayed nonreactive morphologies.

Treatment of contusion injured spinal cord with S-220-loaded hydrogel. For all in vivo experiments, young male adult Wistar rats weighing between 180 and 200 g were purchased from Envigo. The skin and muscle overlying the spinal column were incised, and a laminectomy was performed between the T9 and T10 levels. Following fixation of the adjacent $\mathrm{T} 9$ and $\mathrm{T} 11$ vertebral body to suspend the target region, a standardized moderate (12.5 $\mathrm{mm}$ ) thoracic spinal contusion was inflicted at T10 thoracic vertebral level by the use of a NYU impactor device (Mascis rat model III; Rutgers University) as previously described (Young, 2002; Kjell and Olson, 2016). Following the injury, muscle layers were sutured and the skin was closed. Postinterventional care included manual voiding of the bladder twice a day until voiding reflex was regained. Three weeks after the induction of the injury, rats were randomly allocated to treatment groups. For rats receiving treatment, a $10 \mu \mathrm{l}$ Hamilton syringe was loaded with the pregel format of the hydrogel using RN 26S gauge needle (26S/102/pst2/tapY) (Hamilton; Supelco). The Hamilton syringe was mounted on a stereotaxic frame and armed into $45^{\circ}$ angle. The dura was opened, and the needle was introduced into the dura with the fine controllers. A maximum of $3 \mu \mathrm{l}$ was injected at $1 \mu \mathrm{l} / \mathrm{min}$. The needle was left in place for $10 \mathrm{~min}$ after injection to prevent the leakage of the pregel solution. The dura was covered with a piece of saline-soaked gel foam, muscle layers were sutured and the skin was closed. Buprenorphine ( $0.3 \mathrm{mg} / \mathrm{kg}$; Vetergesic; Alstoe Animal Health) was given subcutaneously for the management of acute pain after surgery and saline $(0.9 \% \mathrm{NaCl}, 5 \mathrm{ml})$ was administered subcutaneously to compensate for any blood loss. Locomotor function was assessed by two observers independently using the 21-point Basso, Beattie \& Bresnahan (BBB) open field locomotor scale on postoperative days $1,3,14,21,28,35,42$, and 49 .
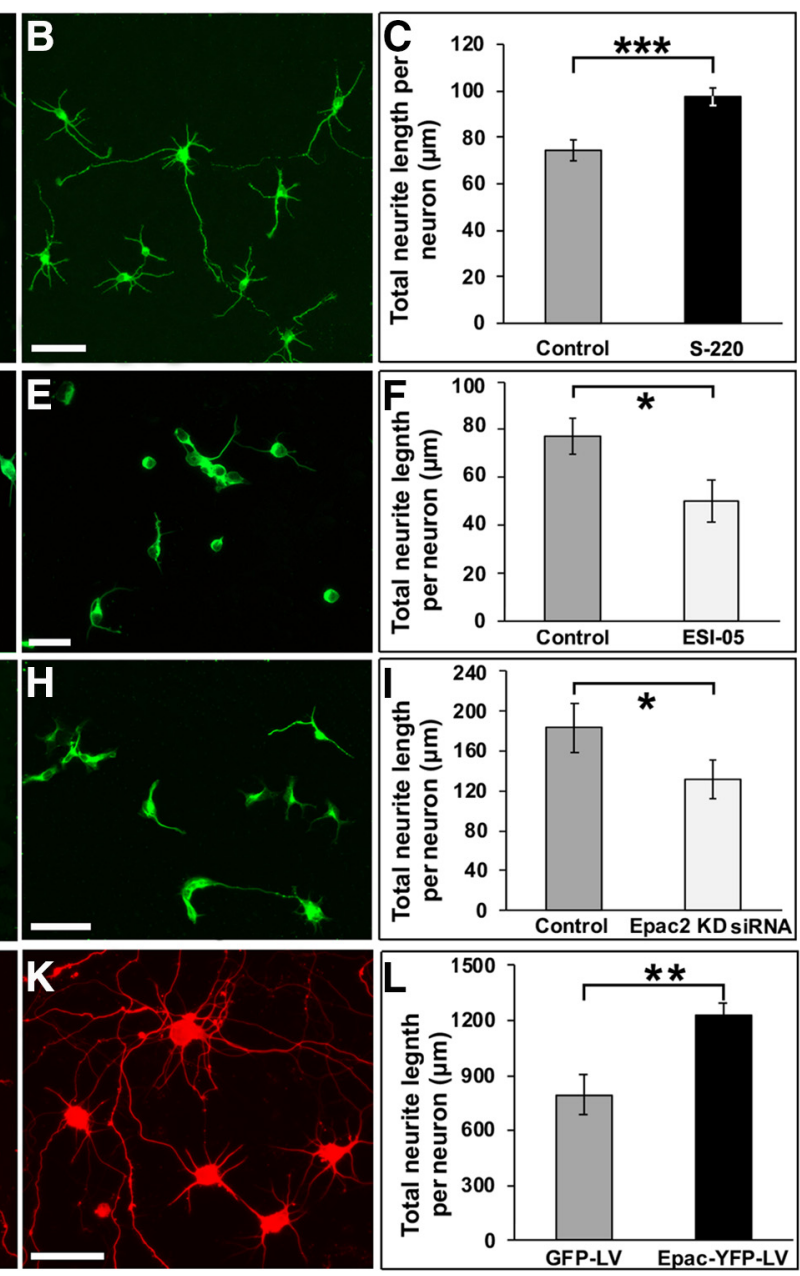

Figure 1. The effects of Epac2 modulation on cortical neurite outgrowth. $\boldsymbol{A}-\boldsymbol{C}$, Epac2 agonist $\mathrm{S}-220$ promoted significant neurite outgrowth. $\boldsymbol{A}$, Control. $\boldsymbol{B}$, Treated with S-220. C, Quantification of total neurite length shows that S-220-treated eurons had significantly longer neurites. $\boldsymbol{D}-\boldsymbol{F}$, Epac2 antagonist ESI-05 significantly decreased cortical neurite outgrowth Ditly shorter neurites. G-I, siRNA knockdown of Epac2 significantly decreased cortical neurite outgrowth. G, Scrambled

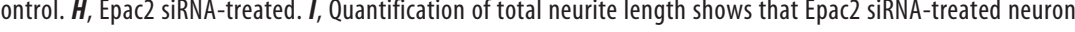
growth. J, LV/GFP-transduced neurons. $K$, LV/Epac-YFP-transduced neurons. $L$, Quantification of total neurite length shows that LV/Epac-YFP-transduced neurons had significantly longer neurites. $\boldsymbol{A}, \boldsymbol{B}, \boldsymbol{D}, \boldsymbol{E}$, Cultures were grown for $24 \mathrm{~h} . \boldsymbol{G}$, $\boldsymbol{H}$, Cultures were grown for $48 \mathrm{~h}$. J, $\boldsymbol{K}$, Cultures were grown for $7 \mathrm{~d}$ to allow enough time for gene expression. All cultures were immunostained for $\beta$-tubulin-III. Unpaired Student's $t$ test: ${ }^{*} p<0.05 ;{ }^{* *} p<0.01 ;{ }^{* * *} p<0.001 . n=3 /$ group Data are mean \pm SEM. Scale bars: $\boldsymbol{A}-\boldsymbol{E}, \boldsymbol{J}-\boldsymbol{K}, 50 \mu \mathrm{m} ; \boldsymbol{G}, \boldsymbol{H}, 100 \mu \mathrm{m}$.

Statistical analysis. Sigma-Aldrich Plot 13.0 and Origin Pro 2018b software was used for all statistical analyses. The normality test Shapiro-Wilk was performed to ensure normal distribution of the data and suitability of parametric tests. Equal variance was tested using the Brown-Forsythe test. Statistical analyses were performed using unpaired Student's $t$ test and one-way ANOVA, using Bonferroni's multiple-comparison test for post hoc analysis. If normality was not met, nonparametric tests were used: the Mann-Whitney rank sum test and the Kruskal-Wallis one-way ANOVA on ranks with Dunn's method or Tukey test post hoc correction. For in vitro studies, all data represent mean \pm SEM.

For ex vivo studies, between 4 and 6 replicates were done per condition and experiment and are represented by a circle in the quantification graph. All values quoted are expressed as the mean \pm SEM (box limits) \pm 5\%-95\% (error bars). Two-way repeated-measure ANOVA was performed for locomotor recovery (BBB scale) analysis, followed by Bonferroni's post hoc multiple-comparison adjustment to calculate the significant levels. "Treatment" is a between-subjects variable, whereas "time" is a within-subjects variable. The in vivo data were presented as 

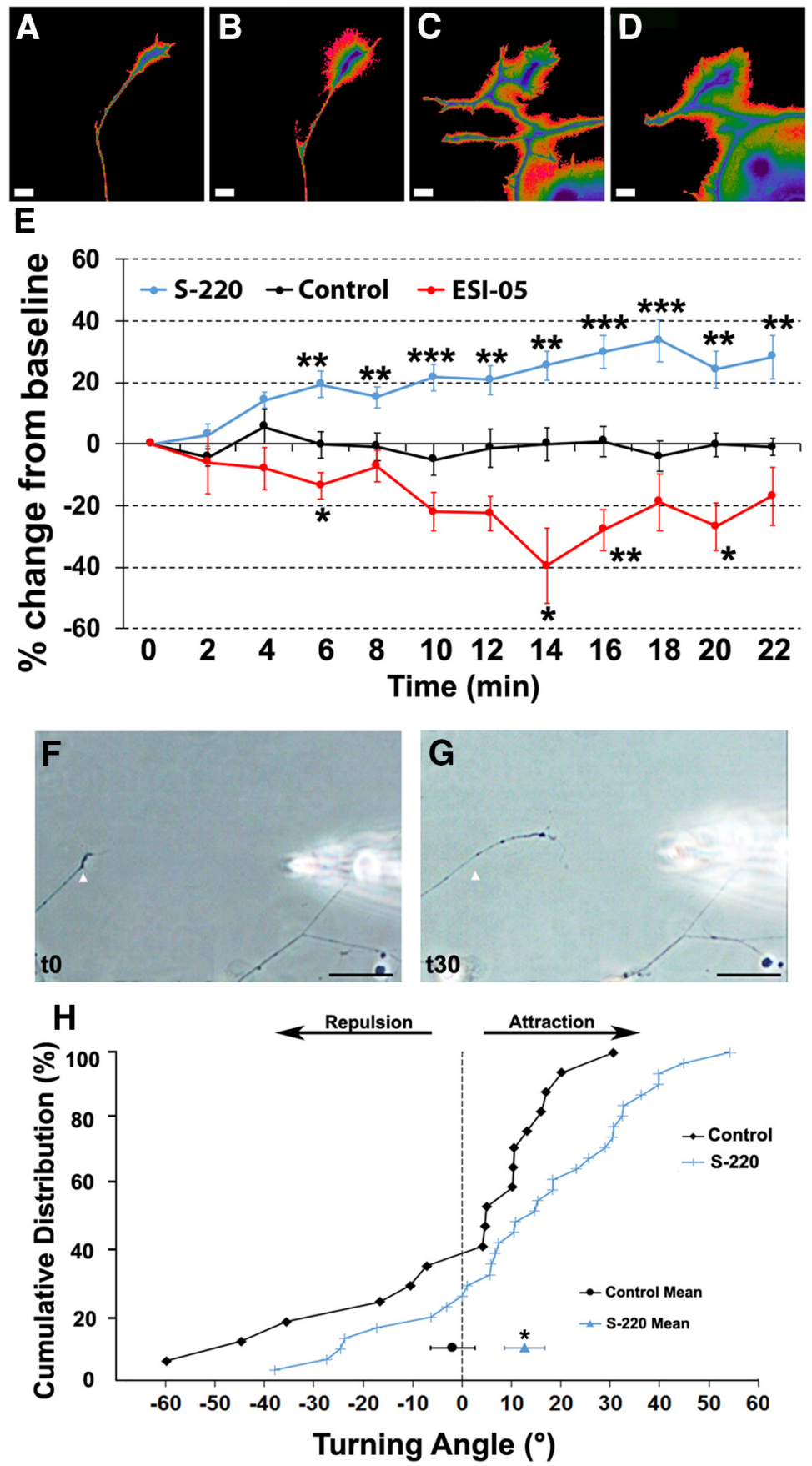

Figure 2. Epac agonist activates Epac protein in DRG growth cones and induces growth cone attraction. $\boldsymbol{A}-\boldsymbol{D}$, Representative images of FRET SE before $(\boldsymbol{A})$ and after $(\boldsymbol{B})$ the addition of the Epac2 agonist S-220 and before $(\boldsymbol{C})$ and after $(\boldsymbol{D})$ the addition of the Epac2 antagonist ESI-05. E, FRET SE measured over time shows a significant activation and inactivation of Epac after the addition of the S-220 (blue) and ESI-05 (red), respectively, compared with control (black) $(n=4) . \boldsymbol{F}, \boldsymbol{G}$, Representative images of DRG growth cones turning toward a gradient of $S-220$ at time $0(\boldsymbol{F})$ and $30 \mathrm{~min}(\boldsymbol{G})$. $\boldsymbol{H}$, Cumulative frequency plots of turning angles show a clear switch toward the right in gradients of S-220 (blue) compared with control F12 (black), indicating greater attraction toward the direction of Epac2 activity. Average turning angles are shown above the abscissa $(n=10)$. Data are mean \pm SEM. Scale bars: $\boldsymbol{A}-\boldsymbol{D}, 10 \mu \mathrm{m} ; \boldsymbol{F}, \mathbf{G}, 50 \mu \mathrm{m} .{ }^{*} p<0.05 ;{ }^{* *} p<0.01 ;{ }^{* *} p<0.001$.

mean \pm SEM. All statistical analyses were performed using Origin Pro $2018 \mathrm{~b}$ and SigmaPlot 14.0 software.

\section{Results}

The elevation of Epac2 activity enhances neurite outgrowth in vitro

Axonal repair following SCI requires the restoration of relays from higher brain centers, such as corticospinal tracts, as well as ascending sensory pathways relaying information from the periphery to the brain. We have expertise in model systems involving cortical neurons and DRG neurons and have used both neuronal types in the current study.

Using cultured postnatal rat cortical neurons, we found that neurite outgrowth (i.e., mean total neurite length per neuron) was significantly enhanced by the treatment of S-220 at $2.5 \mu \mathrm{M}$ for $24 \mathrm{~h}$, compared with that of the controls (agonist vs control: $97.5 \pm 4.1$ vs $72.8 \pm 2.6$ $\mu \mathrm{m}, p=0.0002$, unpaired Student's $t$ test; Fig. $1 A-C)$. Moreover, neurite outgrowth was significantly inhibited by bath application of ESI-05 at $10 \mu \mathrm{M}$ for $24 \mathrm{~h}$ in culture (antagonist vs control: $50.1 \pm 4.8$ vs $77.8 \pm 4.8 \mu \mathrm{m}, p=0.018$, unpaired Student's $t$ test; Fig. $1 D-F$ ). Furthermore, we used genetic tools, such as siRNA and LVs, to illustrate the importance of Epac for neuronal growth. Thus, cortical neurons subjected to specific Epac2 siRNA knockdown for $48 \mathrm{~h}$ showed significantly shorter total neurite length in comparison with that of the controls (siRNA vs control: $131.4 \pm 19.9$ vs $183.3 \pm 24.3 \mu \mathrm{m}, p=$ 0.018 , unpaired Student's $t$ test; Fig. $1 G-$ $I)$. Similar results were also obtained for postnatal rat DRG neurons when they were treated with the S-220 (S-220 vs control: $991.9 \pm 202.9$ vs $399.2 \pm 101.4 \mu \mathrm{m}$, $p=0.005787$, unpaired Student's $t$ test; data not shown) or when they were transfected with Epac2 siRNA (siRNA vs control: $889.7 \pm 49.9$ vs $1677.9 \pm 105.6 \mu \mathrm{m}$, $p=1.1 \times 10^{-11}$ unpaired Student's $t$ test; data not shown). Cortical neurons were transduced with LVs (96.7 $\pm 3.4 \%$ transfection efficiency) and left to grow for $7 \mathrm{~d}$ to ensure protein expression. Neurons transfected with LV/Epac-YFP had significantly more neurite outgrowth compared with that of LV/GFP-transduced neurons (LV/Epac-YFP vs LV/GFP: $1229.2 \pm 20.0$ vs $795.2 \pm 110.4 \mu \mathrm{m}, p=$ 0.003 , unpaired Student's $t$ test; Fig. $1 J-L$ ).

Epac2 agonist activates Epac protein in DRG growth cones and attracts growth cones

To demonstrate the activation/deactivation of Epac protein by the soluble agonist S-220 and antagonist ESI-05 in neuronal growth cones, we performed FRET SE microscopy, which allowed the visualization of protein dynamics in situ (Tucker, 2014). DRG neurons were transfected with mTurqDel-EPAC (dDEPCD) cp173Venus (d)-Venus (d) $(\sim 40 \%$ transfection efficiency) and allowed to grow for $48 \mathrm{~h}$ before the experiments were performed. We found that there was a significant increase of Epac activation in growth cones during the 30 min when the S-220 was applied (S-220 vs baseline, $p=8 \times 10^{-8}$, 

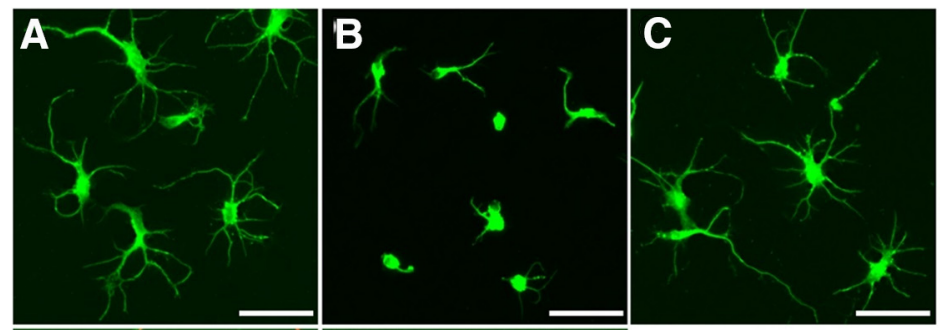

D Total neurite length per neuron
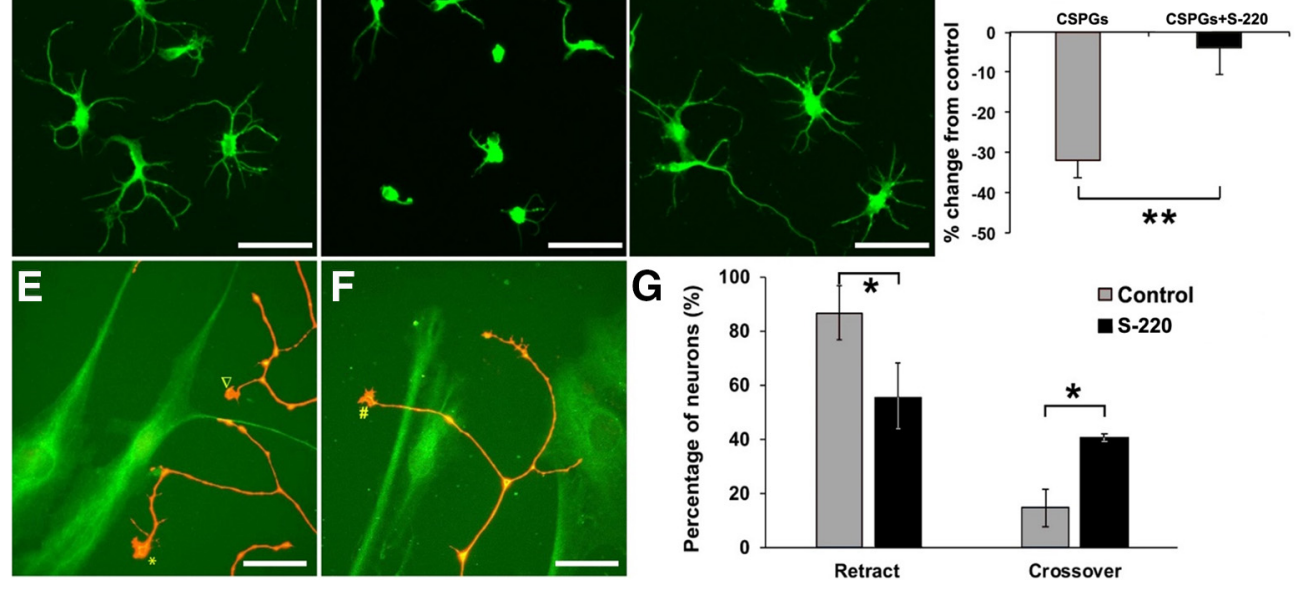

Figure 3. The Epac2 agonist overcomes inhibitory environments for cortical neuron growth. $\boldsymbol{A}, \boldsymbol{\beta}$-tubulin-III-positive cortical neurons grew neurites on PDL-coated coverslips. $\boldsymbol{B}$, Cortical neurons treated with CSPGs showed significantly shorter neurite lengths compared with control. C, Epac2 agonist S-220 attenuated the inhibitory effect of CSPGs on cortical neurite outgrowth. D, Quantification represents the percentage of change from control and shows a significant reduction in inhibition by CSPGs when S-220 was simultaneously applied. Cultures were grown for $48 \mathrm{~h}$. $E-G$, $S-220$ also showed the effect in overcoming the astrocyte inhibition. Three neurite growth cone behaviors of DRG neurons cocultured with mature astrocytes were observed: retract $(\boldsymbol{E}, \nabla)$, reflect $\left(\boldsymbol{E}^{*}\right)$, and crossover $(\boldsymbol{F}, \#)$ using time-lapse live cell microscopy. Green color $(\mathrm{E}, \mathrm{F})=\mathrm{GFAP}$ staining; Red color $(\mathrm{E}, \mathrm{F})=\beta$-tubulin-lll staining. $\boldsymbol{G}$, Quantification showed a significant reduction in the retract/reflect behaviors of neurites and a significant increase in the crossover behavior of neurites in cells treated with the Epac 2 agonist compared with control.D, G, Mann-Whitney Rank Sum test: ${ }^{*} p<0.05 ;{ }^{* *} p<0.01 . n=3$ /group. Data are mean \pm SEM. Scale bars: $\boldsymbol{A}-\boldsymbol{C}, 50 \mu \mathrm{m} ; \boldsymbol{E}, \boldsymbol{F}, 25 \mu \mathrm{m}$.
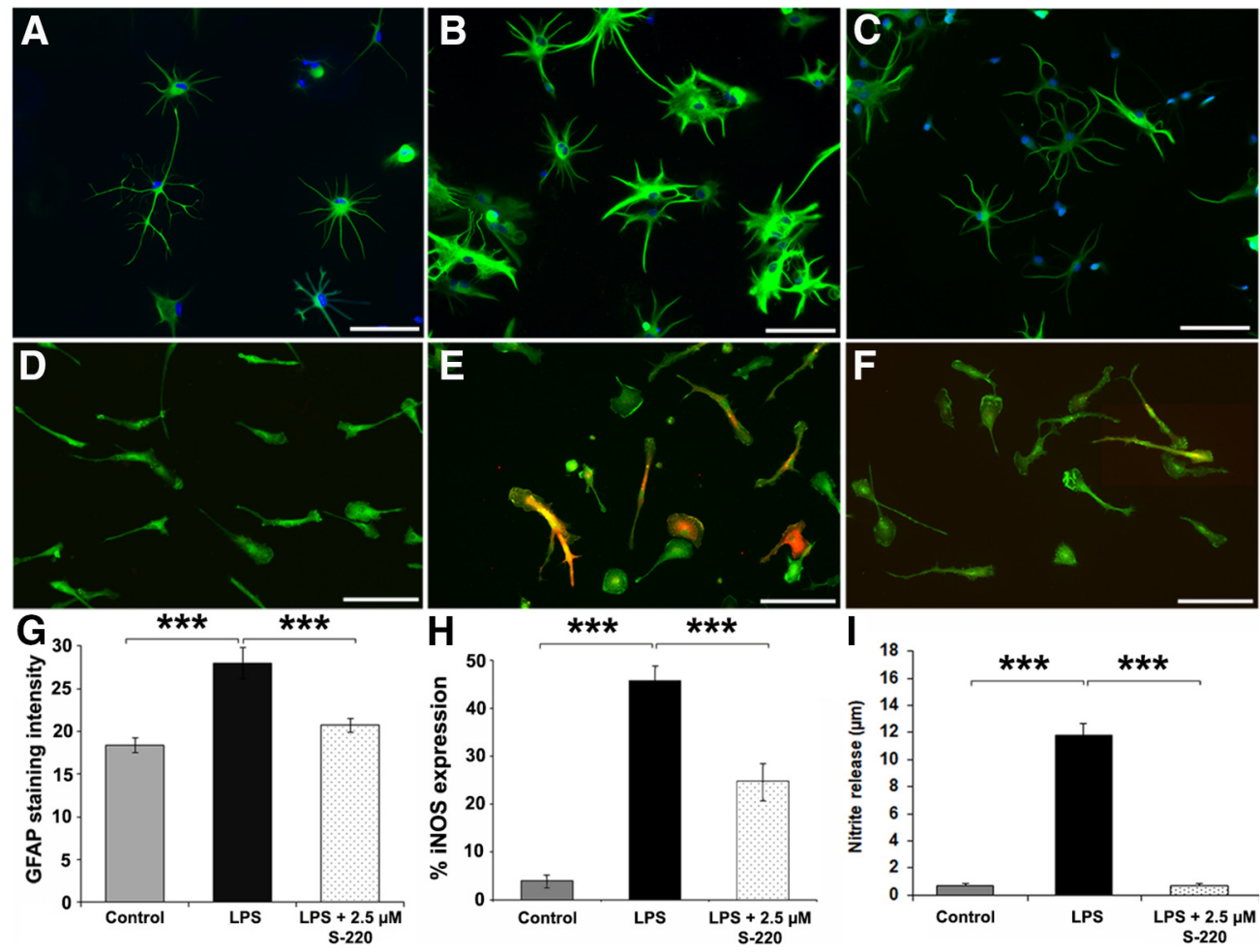

Figure 4. Epac2 agonistS-220 attenuates LPS-induced astrocyte and microglial activation in vitro. Representative fluorescent images of control $(\boldsymbol{A}), \mathbf{L P S}$-treated $(\boldsymbol{B})$, and LPS $+\boldsymbol{S}$-220-treated $(\boldsymbol{C})$ astrocytes (immunostained for GFAP). Representative fluorescent images of control (D), LPS-treated (E), and LPS + S-220-treated $(\boldsymbol{F})$ microglia immunostained for Iba-1 (green) and iNOS (red). $\boldsymbol{G}$, Quantification of the mean fluorescence intensity of GFAP showed significant difference between control and LPS-treated astrocytes, and between LPS and LPS + S-220-treated astrocytes. $\boldsymbol{H}$, Quantitative image analysis showing significant differences in iNOS-immunoreactive cell numbers among control, LPS-treated, and LPS + S-220-treated microglia.I, The Griess assay demonstrated a significant increase of nitrate concentration in the supernatant collected from LPS-treated microglial cultures compared with that of the control. Data are mean \pm SEM. $n=3 /$ group. One-way ANOVA with Bonferroni post hoc test: ${ }^{* *} p<0.001$. Scale bars, $100 \mu \mathrm{m}$.

unpaired Student's $t$ test; Fig. $2 A, B, E)$. Conversely, when ESI-05 was applied, we found significantly reduced Epac activation in the growth cones compared with the baseline (ESI-05 vs baseline Epac2, $p=0.001$, unpaired Student's $t$ test; Fig. $2 C, D, E)$. Using the growth cone turning assay, we examined the effect of asymmetric Epac2 activation on neonatal rat DRG neurites by creating a gradient of the S-220 agonist. Figure $2 H$ showed that neonatal DRG growth cones displayed a random response to a gradient of 

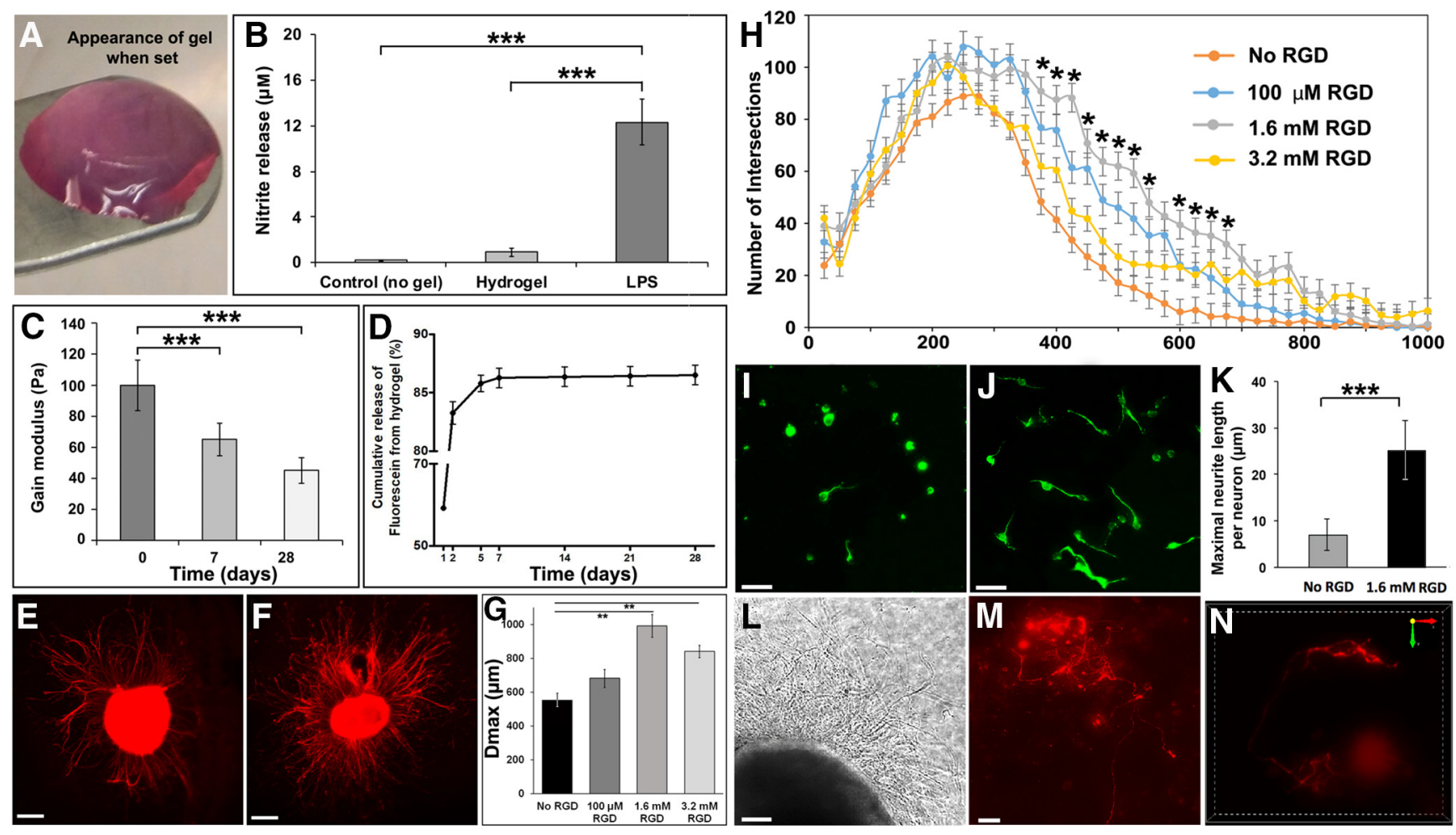

Figure 5. The hydrogel degrades gradually, induces minimal immune response, and supports marked neurite outgrowth in 2-dimentional cultures. $\boldsymbol{A}$, An image of a freshly prepared gel. $\boldsymbol{B}$, The Griess assay showed low levels of nitrite in gel culture media compared with that of LPS-exposed primary microglia cultures. $C$, Gel stiffness showed a gradual decrease in gain modulus from the linear region of the raw data $(0-100 \mathrm{~Hz}$, rheology). $\boldsymbol{D}$, In vitro cumulative percentage drug release versus time profile. Optimization of RGD concentration to promote neurite outgrowth of DRG explants in two dimensions. E, DRG explant in gel without RGD.F, DRG explant in $1.6 \mathrm{~mm}$ RGD gel. G, Quantification of maximal distance of neurite outgrowth showed significant differences at 1.6 and $3.2 \mathrm{~mm}$ RGD compared with control. $\boldsymbol{H}$, Quantification of neurite densities of DRG explants at different concentrations of RGD. I, Cortical neurons on gel without RGD. J, Cortical neurons on gel with $1.6 \mathrm{~mm}$ RGD. $K$, Quantification of neurite length in gels with and without RGD, showing significantly enhanced neurite outgrowth on gels with $1.6 \mathrm{~mm} R G D$. $L$, DRG explants grew extensive neurites in three dimensions within the gel with $1.6 \mathrm{~mm} R G D$ at $72 \mathrm{~h}$ after plating. $M$, Z-stack image of DRG neurons growing in three dimensions within the $1.6 \mathrm{~mm} R G D$ gel. $N, Z$-plane view of DRG neurons. DRG explants were immunostained for GAP-43 $(\boldsymbol{E}, \boldsymbol{F})$. DRG neurons and cortical neurons were immunostained for $\beta$-tubulin- $I I I(I, J, M, N)$. Data are mean \pm SEM. $n=3 /$ group. Scale bars: $\boldsymbol{E}, \boldsymbol{F}, 200 \mu \mathrm{m}$; $\boldsymbol{I}, \boldsymbol{J}, \boldsymbol{L}, 100 \mu \mathrm{m} ; \boldsymbol{M}, 50 \mu \mathrm{m}$. One-way ANOVA with Bonferroni's posthoc $(\boldsymbol{B}, \boldsymbol{D}, \boldsymbol{G}, \boldsymbol{H})$, Kruskal-Wallis ANOVA on Ranks with Tukey posthoc $(\boldsymbol{C})$, and unpaired Student's test $(\boldsymbol{K}):{ }^{*} p<0.05$; ${ }^{* *} p<0.01$; ${ }^{* * *} p<0.001$.

F12 culture media emitted from a micropipette placed at a $45^{\circ}$ angle to the initial orientation of the projecting growth cones, with a mean turning angle of $-1.92 \pm 0.05^{\circ}$ at $30 \mathrm{~min}$. In contrast, growth cones displayed a strong chemoattraction when S-220 was emitted from the pipette (Fig. $2 F, G$ ), and the mean turning angle of $12.7 \pm 4.1^{\circ}$ at $30 \mathrm{~min}$ was significantly greater than that of F12 culture media ( $p=0.045$, unpaired Student's $t$ test; Fig. 2H).

\section{The elevation of Epac2 activity overcomes inhibitory effects on cortical neuron growth in vitro}

To further explore the potential of Epac2 activation as a promising strategy to promote axonal outgrowth in the post-SCI environment, we cultured postnatal rat cortical neurons together with CSPGs, which significantly inhibited the outgrowth of cortical neurons by $32.1 \pm 8.7 \%$ compared with the controls $(p=$ 0.003, Mann-Whitney Rank Sum test; Fig. $3 A, B, D)$. S-220 treatment was able to overcome the inhibitory effect of CSPGs on the mean total neurite length per cortical neuron (CSPGs + S-220 vs CSPGs: $32.1 \pm 8.7 \%$ vs $8.7 \pm 3.3 \%, p=0.007$, Mann-Whitney Rank Sum test; Fig. $3 B-D)$. We then analyzed the response of the growth cones of DRG neurons when they encountered inhibitory mature astrocytes. This assay was performed in DRG neurons as it was optimized for this primary neural type. The growth cones had the choice of either growing over astrocytes or remaining on the laminin substrate during a period of $30 \mathrm{~min}$ time lapse.
Growth cone response behaviors were classified into two categories: retract/reflect and crossover, respectively (Fig. $3 E, F$ ). When S-220 was added to the cocultures, we found a significantly increased proportion of growth cones growing over astrocytes compared with that of the controls (S-220 vs control: $45.7 \pm$ $10.3 \%$ vs $11.2 \pm 4.2 \%, p=0.0216$, unpaired Student's $t$ test; Fig. $3 G)$. Conversely, we found a significant reduction in the proportion of retracting/reflecting growth cones (S-220 vs control: $54.3 \pm 10.8 \%$ vs $88.8 \pm 8.7 \%, p=0.0306$, unpaired Student's $t$ test; Fig. $3 G)$.

\section{The elevation of Epac2 activity by S-220 modulates} LPS-activated astrocytes and microglia

To assess whether S-220 has an effect in preventing and/or modulating the activation of the astrocytes and microglia in vitro, we performed a simultaneous treatment study in which S-220 was given at the same time with $10 \mu \mathrm{g} / \mathrm{ml}$ LPS. Previous experiments in the laboratory demonstrated that the treatment of $10 \mu \mathrm{g} / \mathrm{ml}$ LPS for $4 \mathrm{~h}$ allowed rapid activation of the microglia and astrocytes cells without inducing apoptosis (Georgieva, 2018).

The mean GFAP expression of astrocytes treated with LPS (Fig. 4B) was increased compared with controls (Fig. 4A) (LPS vs control: $27.9 \pm 1.8$ vs $18.4 \pm 0.9, p=3 \times 10^{-6}$, one-way ANOVA, Bonferroni's post hoc; Fig. $4 G$ ). The simultaneous treatment of LPS and S-220 (Fig. 4C) showed a significant reduction of GFAP intensity compared with LPS-treated astrocytes (LPS + 
S220 vs LPS: $20.7 \pm 0.8$ vs $27.9 \pm 1.8, p=$ $2 \times 10^{-4}$, one-way ANOVA, Bonferroni's post hoc; Fig. 4G), being not significantly different from that of the control ones (LPS + S220 vs control: $20.7 \pm 0.8$ vs $18.4 \pm 0.9, p=0.57$, one-way ANOVA, Bonferroni's post hoc; Fig. 4G). Astrocyte morphology was different within groups. LPS-treated astrocytes (Fig. 4B) became reactive, characterized by hypertrophic processes, broader cell bodies, and tend to cluster together. When astrocytes were simultaneously exposed to LPS and S-220 (Fig. 4C), they preserved nonreactive morphology with slender processes similar to that of the control (Fig. 4A).

Microglia reactivity was quantified as the percentage of iNOS marker immunoreactivity. The percentage of iNOS expression of microglia treated with LPS (Fig. 4E) was increased compared with controls (Fig. 4D) (LPS vs control: $45.8 \pm$ 2.9 vs $4 \pm 1.3, p=1.5 \times 10^{-16}$, one-way ANOVA, Bonferroni's post hoc; Fig. $4 H$ ). The simultaneous treatment of LPS and S-220 (Fig. $4 F$ ) showed a significant reduction of percentage of iNOS expression compared with LPS-treated microglia $\left(\right.$ LPS + S220 vs LPS: $24.7 \pm 3.8$ vs $45.8 \pm 2.9, p=3.4 \times 10^{-6}$, one-way ANOVA, Bonferroni's post hoc; Fig. $4 H$ ), being also significantly different from that of the control ones (LPS + S220 vs control: $24.7 \pm 3.8$ vs $4 \pm 1.3, p=6.1 \times 10^{-6}$, one-way ANOVA, Bonferroni's post hoc; Fig. $4 H$ ). Moreover, the simultaneous treatment of LPS and S-220 showed a significant reduction of nitrite release compared with LPS-treated microglia (LPS + S220 vs LPS: $0.7 \pm 0.2$ vs $11.8 \pm 0.9, p=0.0002$, unpaired Student's $t$ test; Fig. $4 I$ ).

\section{Fmoc-based hydrogel shows suitable properties for spinal cord repair}

Self-assembling Fmoc-based hydrogel was used as a delivery depot for S-220 agonist and, therefore, its suitability for spinal repair needed to be tested. The hydrogel (Fig. 5A) at $2.5 \mathrm{~mm}$ had a stiffness of $\sim 100 \mathrm{~Pa}$ (Fig. $5 C$ ). We found that the hydrogel degraded gradually in vitro in $\mathrm{PBS}$ at $37^{\circ} \mathrm{C}$, which was manifested by the reduction in gel stiffness $\left(\mathrm{H}_{(2)}=95.27, p=2 \times 10^{-14}\right.$, Kruskal-Wallis ANOVA on Ranks with Tukey post hoc; Fig. 5C) and gel mass over time (one-way $\operatorname{ANOVA}\left(F_{(4,25)}=50.087, p=\right.$ $1.4 \times 10^{-11}$ with Bonferroni's post hoc; data not shown). Minimal activation of gel-exposed microglia was found by measuring nitrite release in culture media, contrasting to the significant nitrite release observed using activating agent LPS alone (Fig. 5B). Using fluorescein, which has a similar molecular weight compared with the Epac2 agonist, we estimated the cumulative release of the agonist from the hydrogel (Perale et al., 2012; Wilems and Sakiyama-Elbert, 2015). The hydrogel showed a two-phase release profile over a $28 \mathrm{~d}$ period (i.e., a quick release during the first $7 \mathrm{~d}$ followed by a minimal gradual release over the next 3 weeks) (Fig. 5D). The hydrogel can also incorporate functional motifs, such as arginine-glycine-aspartate (RGD) peptides to promote cell adhesion (Zhou et al., 2009). Here, we demonstrated that the hydrogel can be functionalized with RGD peptides at different concentrations, with the $1.6 \mathrm{~mm}$ being the
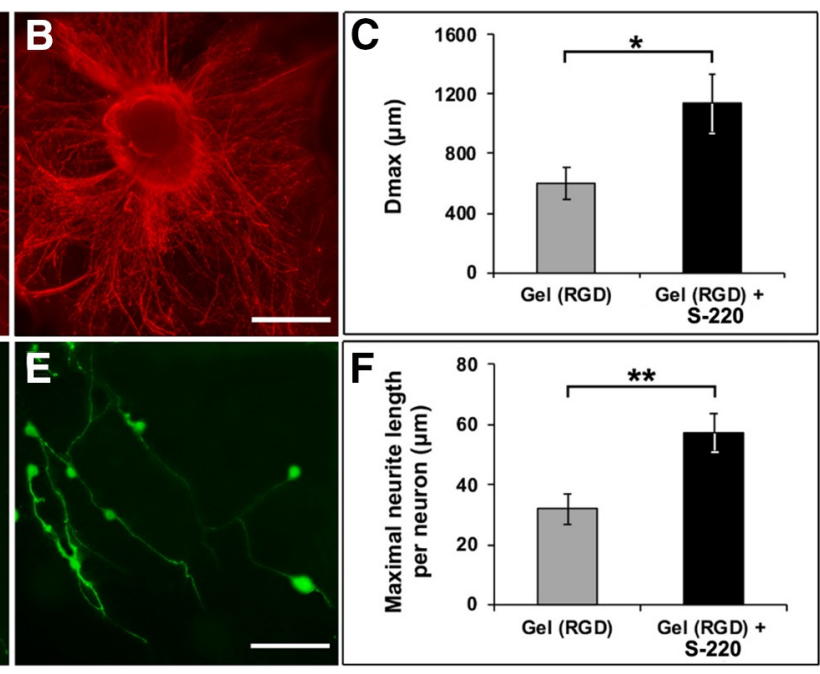

Figure 6. In vitro evidence demonstrating the positive effects of combining the Fmoc hydrogel with Epac2 agonist application on neurite outgrowth. $\boldsymbol{A}-\boldsymbol{C}$, Assessing the effect of combining the Fmoc hydrogel with the Epac2 agonist $\mathbf{S}-220$ in enhancing neurite outgrowth of DRG explants. $\boldsymbol{A}$, Control explant growing in gel alone. $\boldsymbol{B}$, Explant growing in gel in combination with $\boldsymbol{S}-220$. cossing the effect of combining the neurons. $\boldsymbol{D}, \mathrm{DRG}$ neurons growing in gel alone. $\boldsymbol{E}, \mathrm{DRG}$ neurons growing in gel in combination with S-220. $\boldsymbol{F}$, Quantification of group. DRG explants and neurons were immunostained for $\beta$-tubulin-III. Data are mean \pm SEM. Scale bars: $\boldsymbol{A}, 250 \mu \mathrm{m} ; \boldsymbol{B}$ $500 \mu \mathrm{m} ; \boldsymbol{D}, \boldsymbol{E}, 100 \mu \mathrm{m}$. Unpaired Student's $t$ test: ${ }^{*} p<0.05 ;{ }^{* *} p<0.01 . n=3 /$ group.

optimal for neurite outgrowth $\left(\mathrm{D}_{\max }\right)$ of postnatal rat $\mathrm{DRG}$ explants $\left(F_{(3,16)}=7.06, p=0.003\right.$; No-RGD vs $1.6 \mathrm{~mm}$ RGD: $535 \pm$ 3 vs $975 \pm 3 \mu \mathrm{m}, p=0.0035$; No-RGD vs 3.2 mm RGD: $535 \pm 29$ vs $839 \pm 60 \mu \mathrm{m}, p=0.0039$; one-way ANOVA with Bonferroni's post hoc; Fig. $5 E-H$ ). At this optimal concentration of RGD, we observed that the 2-D neurite outgrowth of dissociated postnatal rat DRG neurons (i.e., maximal neurite length per neuron) was comparable with that seen in gels without RGD $(31.8 \pm 5.2$ vs $24.5 \pm 3.8 \mu \mathrm{m}, p=0.137$, unpaired Student's $t$ test; data not shown). However, we found significantly increased 2-D neurite outgrowth of postnatal rat cortical neurons with gels functionalized with $1.6 \mathrm{~mm}$ RGD, compared with that of nonfunctionalized gels $\left(71.4 \pm 9.2\right.$ vs $17.3 \pm 4.3 \mu \mathrm{m}, p=1.2 \times 10^{-6}$, unpaired Student's $t$ test; Fig. $5 I-K)$. Furthermore, we observed that the functionalized hydrogel supported excellent 3-D neurite growth of DRG explants and DRG neurons (Fig. $5 L-N$ ). For simplicity, we refer to the hydrogel functionalized with $1.6 \mathrm{~mm}$ RGD hereafter as "the hydrogel."

\section{The elevation of Epac2 activity enhances 2-D neurite} outgrowth with Fmoc hydrogel in vitro

As a proof of concept, we first explored combination of the Epac2 activation strategy with the gel by adding the S-220 agonist in the media of cultures where DRG explants or DRG neurons grew on the gel surface. We demonstrated that there was a significant increase in neurite outgrowth of DRG explants growing on the gel and simultaneously treated with the agonist compared with that with the gel alone [S-220 + gel vs gel-only: $1141.4 \pm 159.9$ vs $608.3 \pm 93.9 \mu \mathrm{m}\left(D_{\max }\right), p=0.029$, unpaired Student's $t$ test; Fig. $6 A-C]$. In addition, we found that the neurite outgrowth of dissociated DRG neurons was significantly enhanced by combining S-220 and the gel compared with that of the gel alone [S-220 + gel vs gel-only: $57.3 \pm 6.5$ vs $31.9 \pm 5.3 \mu \mathrm{m}$ (mean total neurite length per neuron), $p=0.002$, unpaired Student's $t$ test; Fig. $6 D-F]$. 

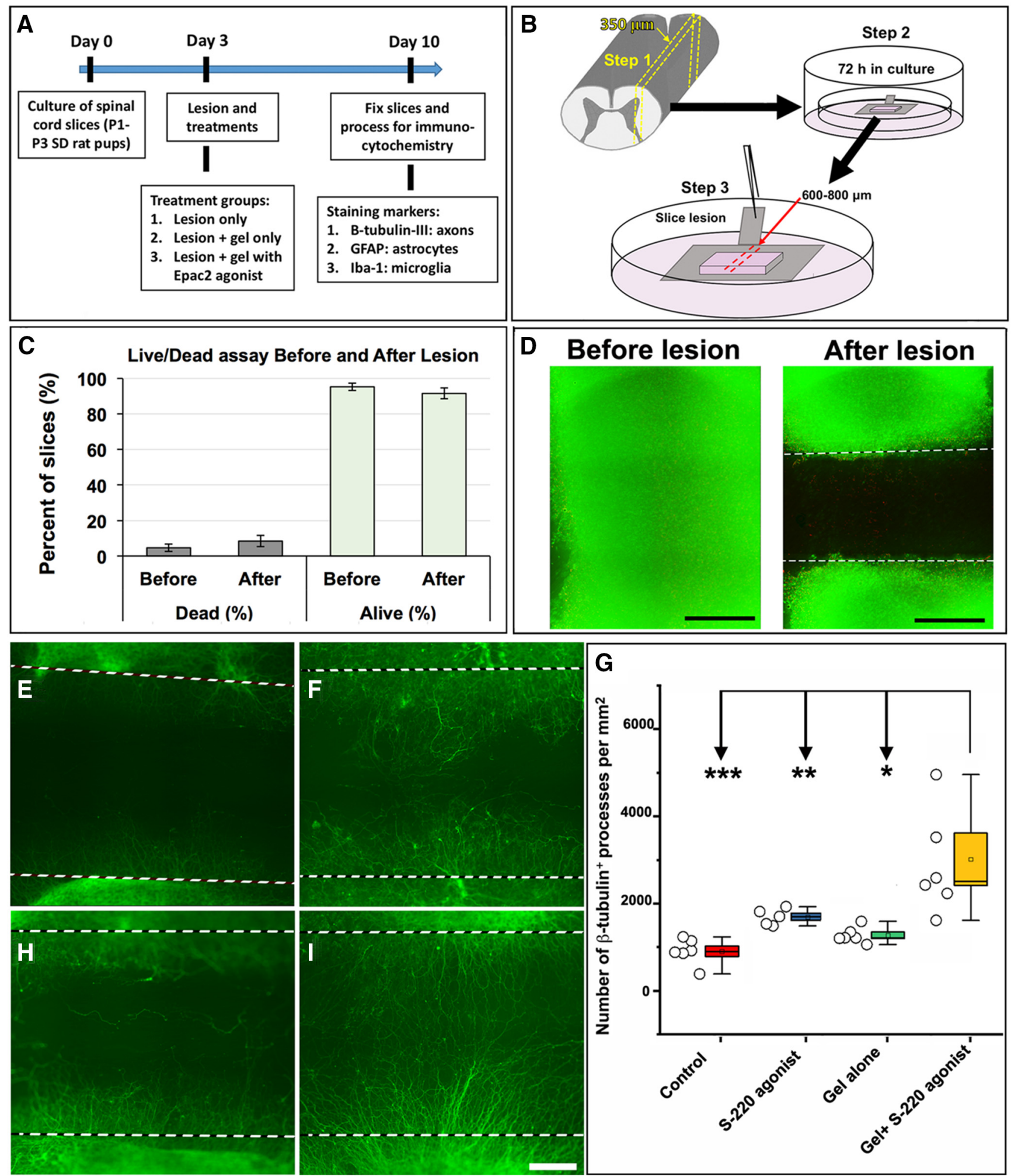

Figure 7. Timeline, study design, and schematic diagram of ex vivo preparation on organotypic spinal cord culture. $A$, Timeline of the experiments. $B$, Schematic diagram depicting the production and lesioning of organotypic spinal cord slice cultures. Yellow dotted lines indicate a spinal cord slice. Red dotted lines and the red arrow indicate a lesion gap. C, Quantification of live/dead assay did not show any difference before and after lesion ( $n=10-15 /$ group). $\boldsymbol{D}$, Representative live/dead-stained fluorescence micrograph of a slice before lesioning and $2 \mathrm{~d}$ after lesion. $\boldsymbol{E}-\boldsymbol{I}$, Using the ex vivo model and $\beta$-tubulin-III staining, we found that, at $7 \mathrm{~d}$ after injury, treatment with either S-220 or hydrogel alone produced similar degrees of neurite outgrowth into the lesion gaps, but significantly more than that of the nontreated slices. $\boldsymbol{E}$, Nontreated slice. $\boldsymbol{F}, \mathbf{S}-220$-treated slice. $\boldsymbol{H}$, Hydrogel-treated slice. $\boldsymbol{I}$, Combination-treated slice. White dashed lines indicate lesion margins. The agonist was added to culture media. $\mathbf{G}$, The axonal profile analysis showed that, when the gel was combined with S-220, there was significantly more axon growth in the lesion gap than those following treatments with S-220 or hydrogel alone. C, Data are mean \pm SEM. G, Data are mean \pm SEM (box limits). Bars above and below each box represent $5 \%$ and $95 \%$ confidence limits. Circles represent individual biological replicates $(n=4-6)$. One-way ANOVA, Bonferroni's post hoc test: ${ }^{*} p<0.05$; ${ }^{* *} p<0.01$; ${ }^{* *} p<0.001$. Scale bars: $\boldsymbol{D}, 500 \mu \mathrm{m} ; \boldsymbol{E}-I, 200 \mu \mathrm{m}$. 

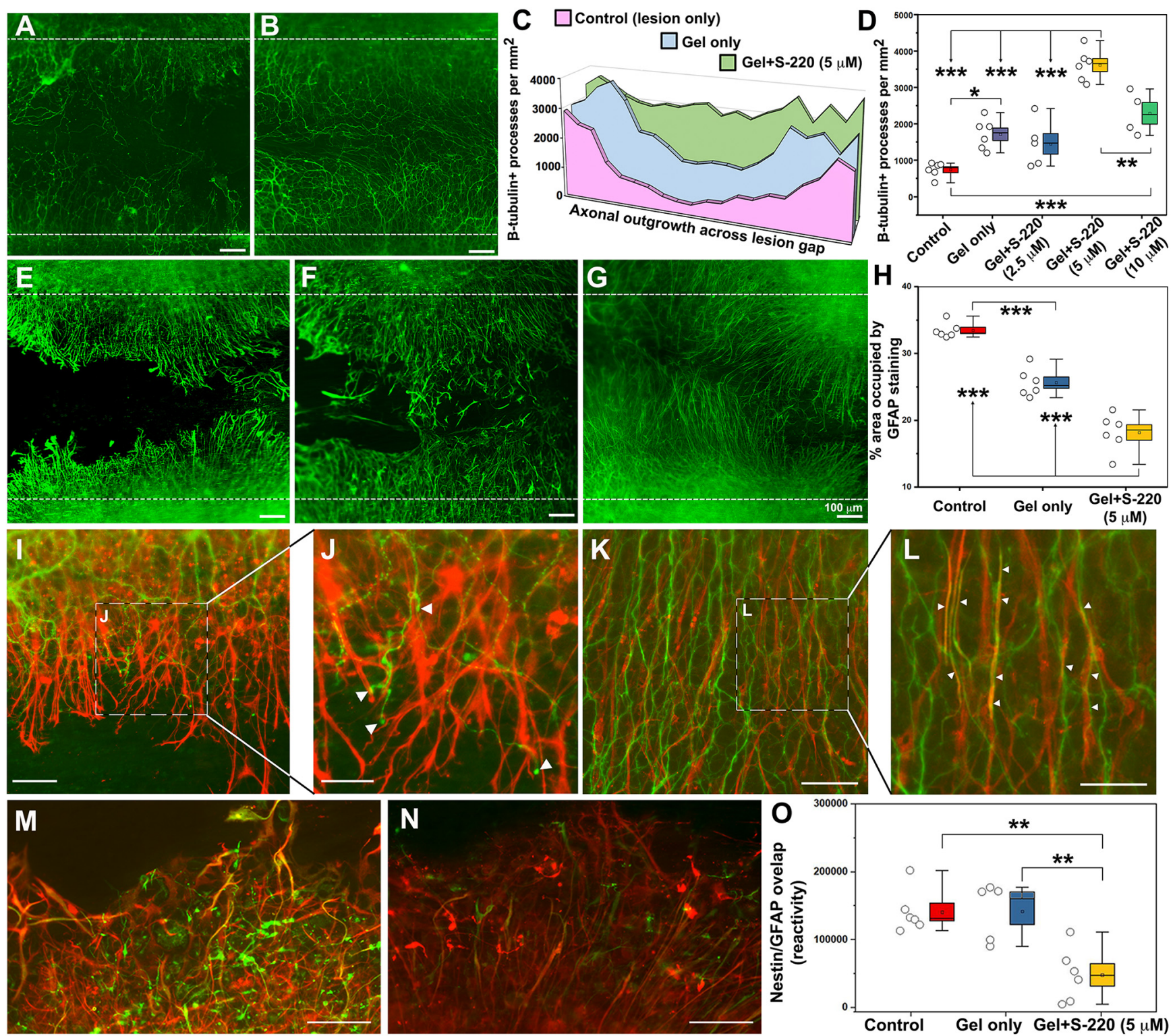

Figure 8. S-220 incorporated into the hydrogel promotes neurite outgrowth and suppresses astrocyte activation. $A, B$, Representative images showing significant difference in neurite outgrowth between gel-only $(\boldsymbol{A})$ and gel $+5 \mu \mathrm{m} S-220(\boldsymbol{B})$. White dashed lines indicate lesion margins. $\boldsymbol{C}$, Neurite growth profiles across the lesion gap, showing a progressive increase with gel-only and gel $+5 \mu \mathrm{M}$ S-220. D, Quantification showing the numbers of $\beta$-tubulin-III ${ }^{+}$processes per square millimeter between control and different concentrations of S-220 delivered in the gel, with $5 \mu \mathrm{m}$ having the greatest effect. $\boldsymbol{E}-\mathbf{G}$, Representative images exhibit astrocyte activation in the lesion using GFAP staining as an astrocyte marker. $\boldsymbol{E}$, Control. $\boldsymbol{F}, \mathbf{G e l}-$ only. $\mathbf{G}, \mathrm{Gel}+5 \mu \boldsymbol{M} \mathbf{S}-220$. $\boldsymbol{H}$, Quantification of the GFAP immunoreactivity intensity showed a significant reduction of mean gray value (optical density) in gel $+5 \mu \mathrm{m} S-220$ compared with the control and the gel-only treatment. $I$, Representative image of the relationship between GFAP (red) and $\beta$-tubulin-III (green) immunoreactive processes in a control injury condition. J, Higher-magnification image showing the collapse of growth cones (white arrowheads) when they meet activated astrocytes. $\boldsymbol{K}$, Representative image of the relationship between GFAP and $\beta$-tubulin-III-immunoreactive processes in a lesion with combined treatment with gel $+5 \mu \mathrm{m} S$-220. L, Higher-magnification image showing the alignment of the astrocytes and $\beta$-tubulin-III-immunoreactive processes (white arrowheads). $\boldsymbol{M}, \boldsymbol{N}$, Levels of astrocyte reactivity were estimated by the overlapping of GFAP (red) and nestin (green). $\boldsymbol{M}$, Representative image of GFAP/nestin reactivity in lesion sites of nontreated slices. $\boldsymbol{N}$, Representative image of GFAP/nestin overlapping in slices treated with S-220 delivered by the hydrogel. $\mathbf{O}$, Quantification of GFAP/nestin pixel overlapping. $\mathbf{D}, \boldsymbol{H}, \mathbf{O}$, Data are mean \pm SEM (box limits). Bars above and below each box represent $5 \%$ and $95 \%$ confidence limits. Circles represent individual biological replicates $(n=4-6)$. One-way ANOVA with Bonferroni's posthoc test: ${ }^{* *} p<$ 0.01; ${ }^{* * *} p<0.001$. Scale bars: $\boldsymbol{A}, \boldsymbol{B}, \boldsymbol{E}-\mathbf{G}, \boldsymbol{M}, \boldsymbol{N}, 100 \mu \mathrm{m} ; \boldsymbol{I}, \boldsymbol{K}, 50 \mu \mathrm{m} ; \boldsymbol{J}, \mathbf{L}, 25 \mu \mathrm{m}$.

The elevation of Epac2 activity promotes axonal outgrowth in an ex vivo model of SCI

To further demonstrate the potential of Epac2 activation for spinal cord repair, we adapted a previously established mouse ex vivo model of SCI using postnatal rat spinal cord organotypic slices (Weightman et al., 2014). The study design, timeline, and schematic diagram of the methodology are shown in Figure $7 A, B$. First, we used a live/dead assay to measure the viability of the slices. The results showed that there was no difference in the percentage of cells labeled by the cell death marker before and after lesioning the slices $(8.4 \pm 3.1$ vs $4.6 \pm 2.1 \%, p=0.148$, unpaired Student's $t$ test; Fig. $7 C, D)$. We then treated the lesioned slices with S-220 being applied to the culture medium alone, or S-220 applied to the culture medium together with hydrogels (not containing S-220) being placed into lesion gaps (Fig. 7E-I). One-way ANOVA showed that there was a significant treatment effect on axonal outgrowth across the lesion gap among different treatment groups $\left(F_{(3,19)}=8.547, p=0.0006\right.$; Fig. $\left.7 G\right)$. Thus, the 

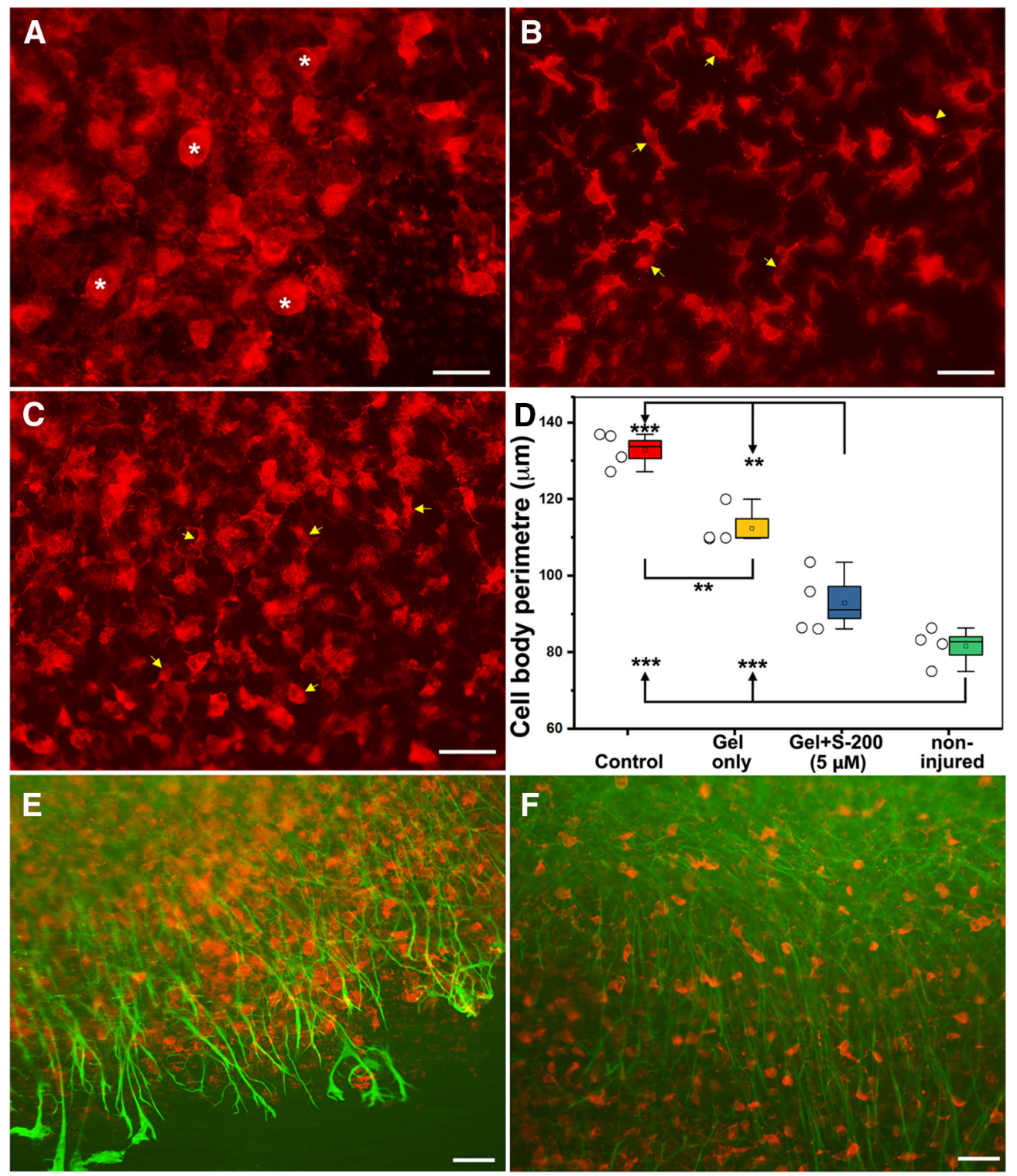

Figure 9. S-220 incorporated into the gel suppresses microglia activation in lesioned spinal cord slice. $\boldsymbol{A}$, Iba-1-immunoreactive cells inside the injury site in control lesion slices. White asterisks indicate activated morphology. $\boldsymbol{B}$, Iba-1-immunoreactive cells inside the injury site in combination-treated slices. Yellow arrowheads indicate resting morphology. $\boldsymbol{C}$, Noninjured microglia. Yellow arrowheads indicate resting morphology. $\boldsymbol{D}$, Cell body perimeter was quantified and used as an indication of activation. Cell body size decreased more significantly in the presence of the hydrogel-only or hydrogel $+5 \mu \mathrm{MS}$-220, respectively. $\boldsymbol{E}$, Representative image of the relationship between GFAP-and Iba-1-immunoreactive cells in an injured control. $\boldsymbol{F}$, Representative image of the relationship between GFAP- and Iba-1-immunoreactive cells in injured slices treated with a combination of hydrogel $+5 \mu \mathrm{m}$ S-220. D, Data are mean \pm SEM (box limits). Bars above and below each box represent $5 \%$ and $95 \%$ confidence limits. Circles represent individual biological replicates $(n=4)$. One-way ANOVA with Bonferroni's posthoc test: ${ }^{* *} p<0.01$; ${ }^{* * *} p<0.001$. Scale bars: $A-C, 25 \mu \mathrm{m} ; E, F, 50 \mu \mathrm{m}$.

results showed that the number of $\beta$-tubulin-III staining profiles per square millimeter per slice was significantly higher in the slices treated with the agonist and the gel compared with those of the control, gel alone, and S-220 alone groups [S-220 + gel vs control, S-220 alone, and gel alone: $3016.5 \pm 596.5$ vs $821.8 \pm$ $102.9(p=0.00055), 1247.9 \pm 73.7(p=0.005)$, and $1694.7 \pm$ $82.9(p=0.0481)$, one-way ANOVA with Bonferroni's post hoc; Fig. $7 G]$.

\section{Epac2 agonist incorporated into Fmoc hydrogel promotes axonal outgrowth}

Our next step was to incorporate S-220 into the gel so that the agonist could be locally released and delivered to the injury site. Since it is a different delivery method, we tested different concen- trations of the agonist to identify the optimal concentration. One-way ANOVA showed that there was a significant concentration effect on axonal outgrowth across the lesion gap $\left(F_{(2,12)}=\right.$ $21.53, p=0.00011$; Fig. $8 D$ ). Thus, the results showed that the number of $\beta$-tubulin-III staining profiles per square millimeter per slice was significantly higher in the slices treated with the gel $+\mathrm{S}-220(5 \mu \mathrm{M})$ compared with those of the gel $+\mathrm{S}-220(2.5 \mu \mathrm{M})$ and gel + S-220 $(10 \mu \mathrm{M})$ [gel + S-220 $(5 \mu \mathrm{M})$ vs gel + S-220 $(2.5$ $\mu \mathrm{M})$ or gel $+\mathrm{S}-220(10 \mu \mathrm{M}): 3613.0 \pm 177.0$ vs $1457.6 \pm 284.6$ $(p=0.000091)$ or $2289.8 \pm 298.2(p=0.0086)$, one-way ANOVA with Bonferroni's post hoc test; Fig. $8 A-D$ ]. Once the optimal concentration of the agonist was established, we then investigated the efficacy of the agonist at this concentration when it was incorporated into the gel in promoting neurite outgrowth 
across the lesion gap. One-way ANOVA showed that there was a significant treatment effect on axonal outgrowth among different experimental groups $\left(F_{(4,22)}=21.14, p=7 \times 10^{-8}\right.$; Fig. $\left.8 A-D\right)$. Thus, the results showed that the number of $\beta$-tubulin-III staining profiles per square millimeter per slice was significantly higher in the slices treated with the gel + S-220 $(5 \mu \mathrm{M})$ compared with those of the control and gel-only groups [gel $+\mathrm{S}-220(5 \mu \mathrm{M})$ vs control or gel-only: $3613 \pm 177$ vs $743 \pm 81.5\left(p=3 \times 10^{-8}\right)$ or $1698.5 \pm 169.4$ ( $p=0.000003)$, Bonferroni's post hoc; Fig. $8 A-D]$.

\section{The elevation of Epac2 activity attenuates the activation of astrocytes in \\ an ex vivo model of SCI}

GFAP staining intensity within the lesion gap was quantified to assess astrocyte reactivity. A marked difference was found in the morphology of the astrocytes across the lesion gap from different experimental groups. GFAP-immunoreactive cells at the forefront into the lesion gap in slices without any treatments were intensively labeled and hypertrophic (Fig. 8E). Slices treated with the gel-only presented a mixed pattern of GFAP-immunoreactive cells (Fig. 8F), whereas those slices receiving S-220 treatment delivered by the gel presented GFAP-immunoreactive cells that were lightly labeled and had elongated polygonal morphologies (Fig. 8G). One-way ANOVA showed that there was a significant treatment effect on GFAP activation across the lesion gap among different experimental groups $\left(F_{(2,15)}=76.79, p=1 \times 10^{-7}\right.$; Fig. $8 H)$. Thus, the results showed that GFAP intensity in the slices treated with the agonist and the gel was significantly lower than those of the control or gel alone groups [ gel + S-220 vs control or gel-only: $18.2 \pm 1.1$ vs $33.5 \pm 0.5\left(p=8 \times 10^{-8}\right)$ or $25.7 \pm 0.9$ $(p=0.00004)$, one-way ANOVA Bonferroni's post hoc test; Fig. $8 H]$. We then performed double staining for $\beta$-tubulin-III and GFAP to study the relationship between astrocytes (hypertrophic and nonhypertrophic) and regrown neurites. In nontreated slices, highly reactive astrocytes appeared to act as a barrier for neurite outgrowth (Fig. 8I). Higher-magnification images demonstrated collapsed growth cones when they confronted highly reactive astrocytes (Fig. $8 \mathrm{~J}$ ). Conversely, in the slices receiving S-220 delivered by the gel, we observed a close relationship between $\beta$-tubulin-III-stained neurite profiles and GFAP-stained astrocyte processes (Fig. $8 \mathrm{~K}$ ). Higher-magnification images demonstrated that regrown axons were indeed aligned to astrocyte processes (Fig. $8 \mathrm{~L}$ ).

Overlapping of nestin and GFAP markers was also quantified as a reactivity parameter as previously described (O'Neill et al., 2017). One-way ANOVA showed that there was a significant treatment effect on nestin/GFAP overlapping across the lesion gap among different experimental groups $\left(F_{(2,16)}=11.6, p=\right.$ 0.0001; Fig. 8O). Thus, the results showed that the mean nestin/ GFAP overlapping pixels were significantly lower in the slices treated with S-220 delivered by the gel, compared with those of the control or gel alone groups [gel + S-220 vs control or gelonly: $48,369.4 \pm 16,194.2$ vs $140,811.6 \pm 12,992.8(p=0.003)$ or $141,925.5 \pm 19,180.4(p=0.002)$, Bonferroni's post hoc; Fig. $8 M-O]$.

\section{The elevation of Epac2 activity attenuates microglial activation in an ex vivo model of SCI}

We used the Iba-1 marker to identify microglia in lesioned slices. We found that, within the lesion site, Iba-1-immunoreactive cells displayed a highly activated morphology (large in cell size and with no processes) in those slices without any treatments (Fig. 9A,

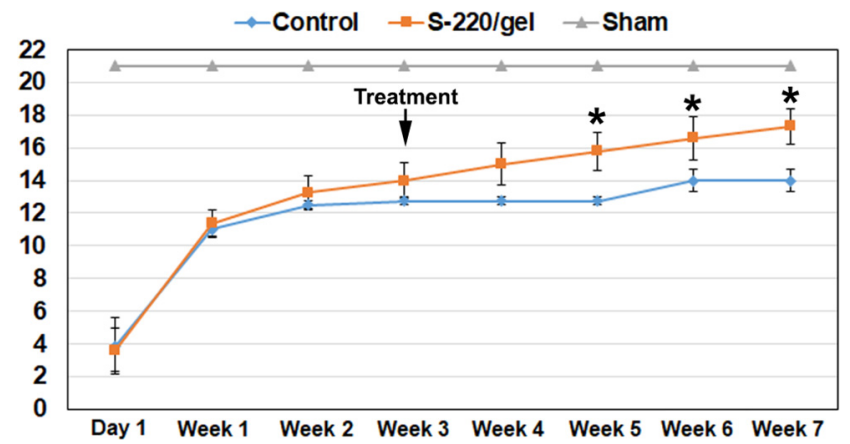

Figure 10. S-220 delivered by Fmoc hydrogel into the lesion of contusion injured spinal cord enhances locomotor functional recovery. Both S-220-treated and nontreated contusion-injured animals showed similar levels of locomotor function at 3 weeks after injury as assessed on the BBB open field task. However, by 2 weeks after treatment (indicated by the black arrow), S-220-treated animals showed significantly better locomotor function (3 points on the BBB scale) compared with the nontreated animals; a difference was maintained until 4 weeks after treatment (the last time point assessed). Data were analyzed with two-way ANOVA followed by Bonferroni's post hoc test: ${ }^{*} p<0.05 . n=4$ or 5 . Data are mean \pm SEM.

white asterisks). This activated morphology was in contrast to those Iba-1-immunoreactive cells located in the main body of the slices away from the lesion margins (Fig. 9C, yellow arrowheads) that exhibited a nonactivated morphology ( small in cell size and with ramified processes). However, we observed that, in those slices treated with S-220 delivered by the gel, Iba-1-immunoreactive cells within the lesion site resembled the nonactivated morphology seen in the main slice body (Fig. 9B, yellow arrowheads). We then performed a quantification to determine the differences in Iba-1-immunoreactive cells within the lesion site among different experimental groups by measuring cell body perimeter, which has been shown to correlate with microglial activation (Kozlowski and Weimer, 2012). Thus, the results showed that the mean cell body perimeter of Iba-1-immunoreactive cells per slice was significantly smaller in the slices treated with S-220 and the gel compared with those of the control or gel alone groups [ gel + S-220 vs control or gel alone: $93 \pm 4.2$ vs $123.7 \pm$ $1.7 \mu \mathrm{m}(p=0.000091)$ or $103.1 \pm 2.3 \mu \mathrm{m}(p=0.0086)$, Bonferroni's post hoc test; Fig. $9 D$ ]. In those slices receiving S-220 delivered by the gel, we found that the mean cell body perimeter of Iba-1-immunoreactive cells was similar to that of nonactivated microglia seen in the main slice body (gel + S-220 vs nonactivated microglia in the main slice body: $93 \pm 4.2$ vs $81.7 \pm 2.4 \mu \mathrm{m}$, $p=0.115$, unpaired Student's $t$ test; Fig. 9D). The relationship between GFAP-immunoreactive and Iba-1-immunoreactive cells was investigated by examining the colabeling of these two markers. In nontreated slices, we observed that highly reactive astrocytes were intermingled with large, amoeboid, and congregated Iba-1-immunoreactive cells (Fig. 9E), demonstrating that both activated glial cells formed a highly inhibitory environment for neurite outgrowth. In contrast, in the slices receiving S-220 delivered by the gel, we observed smaller nonactivated Iba-1immunoreactive cells, which were sparsely spread around elongated polygonal GFAP-immunoreactive cells (Fig. 9F).

\section{S-220 delivered by Fmoc hydrogel promotes functional recovery after a spinal cord contusion injury}

Injured rats treated with S-220 delivered by the gel at 3 weeks after surgery showed significant improvement in the BBB score, already detectable 3 weeks after treatment compared with injuryonly controls, indicating better controlled limb movements after treatment with S-220-loaded gels (Fig. 10). The improvements 


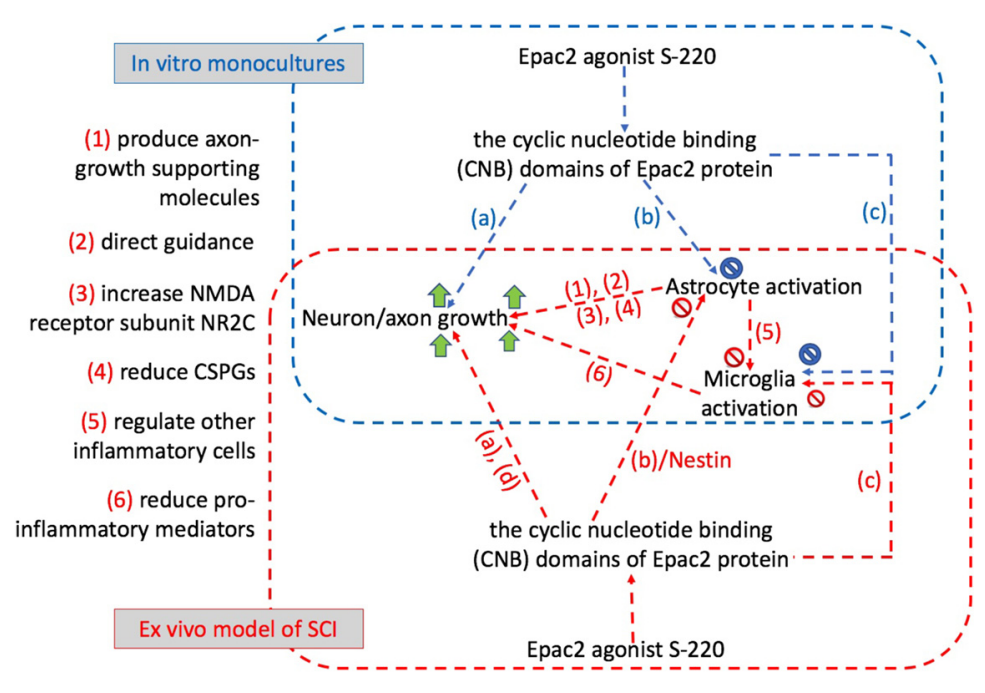

Figure 11. A schematic diagram summarizing the potential mechanisms of Epac2 activation by S-220 in the in vitro and ex vivo studies. Blue dotted rectangle and blue dotted arrows represent the mechanisms for in vitro studies. Red dotted rectangle and red dotted arrows represent the mechanisms for ex vivo studies. Our findings from the in vitro studies with individual monocultures of neurons, astrocytes, and microglia demonstrate that $\mathrm{S}-220$ has direct and individual effects on the following: (a) neurons, promoting their growth possibly via the subsequent activation of Rap 1/B-Raf/ERK signaling pathway (Murray and Shewan, 2008; Murray et al., 2009; Wei et al., 2016); (b) astrocytes, reducing their activation by LPS possibly via the IL-6/STAT3 signaling pathway (Takanaga et al., 2004); and (c) microglia, reducing their activation by LPS possibly via arginase I signaling pathway (Ghosh et al., 2016). S-220 binds to the cyclic nucleotide binding (CNB) domains of Epac2 protein within these cells, triggering downstream signaling pathways (Tucker, 2014). Our findings from the ex vivo studies, however, suggest that S-220 manifests its effects on these cells in a more complex and intermingled manner as follows: (1) elongated astrocytes following S-220 treatment may resemble radial glial progenitors, releasing axon-growth supporting molecules (Garcia et al., 2004); (2) elongated astrocytes following S-220 treatment may provide direct guidance for axonal growth (Anderson et al., 2016; Robichaux and Cheng, 2018); reduced astrocyte activation by S-220 may lead to upregulate the gene for NMDA receptor subunit NR2C, which is a key part in the tripartite synapse regulating astrocyte-neuron communication (Paco et al., 2016); (4) reduced astrocyte activation by S-220 may downregulate the genes responsible for the production of proteoglycans, including CSPGs, thereby reducing their inhibitory effects on axonal outgrowth (Paco et al., 2016); (5) reduced astrocyte activation by S-220 may involve STAT3/nestin, and subsequently may regulate other inflammatory cell behavior, such as that of microglia at the spinal cord lesion site (Wanner et al., 2013); and (6) reduced microglial activation via astrocyte regulation may lead to reduced production of proinflammatory mediators (Steininger et al. 2011), thereby promoting axonal outgrowth. It is also highly likely that $\$-220$ may have direct and individual effects on these three types of cells in the ex vivo model, including transcriptional changes in arginase I, IL-6, secretory leukocyte protease inhibitor, and metallothionein in neurons (d) (Hannila and Filbin, 2008; Siddiq and Hannila, 2015), which have been shown to promote axonal outgrowth and overcome inhibitory molecules. Red and blue small circles with diagonal across represent "stop/inhibit". Green arrows represent "promote/enhance"; ERK = extracellular signal-regulated kinases; STAT3 = signal transducer and activator of transcription 3; IL-6 $=$ interleukin 6; NMDA $=\mathrm{N}$-methyl-D-aspartate.

were sustained for the rest of the study. Two-way ANOVA showed that there were significant treatment effects $\left(F_{(1,35)}=\right.$ $16.17, p<0.001$; Fig. 10). At week 4 after treatment, contused rats that received S-220-loaded gel significantly increased locomotor behavior, reaching an average BBB score of $17.3 \pm 0.9$, compared with $14.3 \pm 1.1$ for injury-only controls ( $p=0.042$, Bonferroni's post hoc analysis; Fig. 10).

\section{Discussion}

Promoting injured adult CNS mammalian neurons to regrow after injury remains a significant challenge in modern medicine. Among different strategies, cAMP and its downstream effectors have been shown to enhance neurons' outgrowth capacity. Here, we demonstrate, for the first time, using pharmacological and genetic tools, that specific elevation of Epac2 activity, a downstream target of cAMP, significantly enhances neurite outgrowth of cultured postnatal DRG and cortical neurons. Moreover, Epac2 activation promotes significant axonal outgrowth in lesioned spinal cord slices in an ex vivo model of SCI. We reveal novel evidence that Epac2 elevation overcomes inhibition on neuron/axon growth by CSPGs and mature astrocytes in vitro and by the postlesion environment of the ex vivo model.
Previously PKA was thought to be solely responsible for cAMP's effects on axonal growth (Siddiq and Hannila, 2015). However, strong evidence suggests that it is Epac, not PKA, that mediates the positive effects of cAMP in promoting axonal growth (Murray et al., 2009; Boomkamp et al., 2014; Wei et al., 2016). Several general agonists were developed to activate Epac, but not PKA, allowing the study of Epac's roles in mediating specific cAMP functions in cell signaling pathways (Enserink et al., 2002). Studies using these agonists demonstrate that Epac mediates cAMP-dependent axonal growth in: (1) embryonic, neonatal, and adult rat DRG neurons (Murray and Shewan, 2008; Murray et al., 2009; Wei et al., 2016); (2) PC12 cells (Christensen et al., 2003); and (3) an in vitro model of SCI and remyelination (Boomkamp et al., 2014). These agonists also regulate axonal elongation in rat embryonic hippocampal neurons (Muñoz-Llancao et al., 2015), and switch the proliferative signal of cAMP to a neuronal differentiation program in $\mathrm{PC} 12$ cells (Kiermayer et al., 2005). However, they failed to discriminate the effects between Epac1 and Epac2.

We previously showed that siRNA knockdown of Epac1 and Epac2 in adult DRG neurons significantly reduced neurite outgrowth (Murray and Shewan, 2008). However, since adult neurons mainly express Epac2, our previous evidence on neurite growth is likely to be mediated solely by Epac2. Here, we used an Epac2 specific agonist (S-220), which has been shown to selectively activate Epac2 (Schwede et al., 2015). We are the first group to report the use of this agonist in primary postnatal rat neurons and spinal cord tissue. Indeed, we used FRET to demonstrate that S-220 enhanced Epac2 protein activity in the growth cones of DRG neurons. Furthermore, we provided direct evidence that DRG neurons turned toward a gradient of S-220, such that Epac2 activation was biased toward the direction of turning. These data convincingly suggest the specificity of this agonist in postnatal neurons. We and others have previously reviewed potential mechanisms of Epac2 signaling in neurite outgrowth (Peace and Shewan, 2011; Batty et al., 2017). Thus, Epac2 is known to activate Rap1, which can subsequently modulate AKT (Mei et al., 2002; Nijholt et al., 2008) and B-Raf-MER-ERK (Qiu et al., 2000; Enserink et al., 2002; Wang et al., 2006) signaling pathways, both being shown to play essential roles in axonal growth and guidance (Liu and Snider, 2001). Epac2 activation may also lead to CREB activation that has been shown to promote neuroplasticity (Wei et al., 2016) (Fig. 11).

Following SCI, there are numerous molecules present at the lesion site, including CSPGs, MAG, Nogo, and OMgp, which are inhibitory to axonal outgrowth (Hannila and Filbin, 2008). A body of evidence has shown that elevation of neuronal cAMP level overcomes the inhibition by MAG and CNS myelin on ax- 
onal outgrowth (Siddiq and Hannila, 2015). The ability of cAMP elevation to overcome inhibition may involve the activation of CREB, which then leads to transcriptional changes in arginase I, interleukin-6, secretory leukocyte protease inhibitor, and metallothionein (Hannila and Filbin, 2008; Siddiq and Hannila, 2015). Upregulation of these genes has been shown to promote axonal outgrowth (Cafferty et al., 2004; Deng et al., 2009; Ghasemlou et al., 2010; Siddiq and Hannila, 2015). Here, we report that activation of Epac2 overcomes inhibition by CSPGs and mature astrocytes on neuronal growth, and this could therefore be mediated through CREB activation. It is important to note that Rap1 can regulate integrins, which are essential for cell adhesion (Bos et al., 2003). Different cell types have been shown to control adhesion to laminin via cAMP-mediated integrin regulation (Murphy et al., 2005). Therefore, Epac2 activation could possibly also lead to Rap1-mediated redistribution and conformational activation of integrins in the growth cone (Bos et al., 2003), thereby modifying their responses to inhibitory molecules in the extracellular matrix after injury (Condic et al., 1999; Tan et al., 2011; Cheah and Andrews, 2018).

Apart from the effects on axonal outgrowth in the ex vivo model, we also report a significantly modified lesion environment consisting of astrocytes and microglia by the Epac2 agonist. Astrocytes form the major cell type that contribute to the formation of the glial scar after SCI, and strong evidence suggests that there is heterogeneity within astrocyte populations in the scar with some cells producing axon-growth supporting molecules (Anderson et al., 2016). The morphology of Epac2 agonisttreated astrocytes in our study remarkably resembles those newly proliferated and elongated astrocytes at the lesion border after SCI, which are regulated by the STAT3 signaling pathway (Wanner et al., 2013) and show similarity with radial glial progenitors providing guidance for neuron growth during CNS development (Garcia et al., 2004). Although the functions of these elongated astrocytes at the lesion border are largely unclear, evidence suggests that they regulate inflammatory cell behavior (Wanner et al., 2013). Here, we demonstrate a similar pattern following S-220 treatment, in that elongated astrocytes exist among Iba- $1^{+}$microglia with nonactivated morphology at the lesion site. It is known that cAMP elevation can cause autocrine interleukin-6 upregulation in astrocytes, which in turn can lead to STAT3 phosphorylation (Takanaga et al., 2004). Moreover, cAMP elevation in astrocytes causes morphological change into a processbearing shape, downregulation of the genes responsible for proteoglycan production, and upregulation of the gene for the NMDA receptor subunit NR2C that is a major component of the tripartite synapse regulating astrocyte-neuron communication (Paco et al., 2016). Therefore, it is conceivable that in our study the S-220 has caused profound changes in astrocytes at the lesion site similar to those described after cAMP elevation and render them supportive for the regrowing axons (Fig. 11). Furthermore, there is evidence that cAMP elevation by a general agonist causes microglial BV-2 cells to increase arginase I production and change to bipolar morphology when cotreated with TNF- $\alpha$ (Ghosh et al., 2016). Epac activation by a general agonist also causes BV-2 cells to reduce their proinflammatory activities (Steininger et al., 2011). Hence, we propose that in our study the S-220 also attenuates the activation of microglia at the lesion site. Together, our data suggest that Epac2 elevation in both astrocytes and microglia may have potently modified the lesion environment from inhibitory to permissive for axonal outgrowth.

With the novel Fmoc-based hydrogel used as a delivery depot for sustained release of S-220, our study confers translational potential. We demonstrate, for the first time, that this hydrogel has excellent biocompatibility and supports excellent neurite outgrowth in vitro and axonal outgrowth in the ex vivo model. Moreover, its stiffness can be tuned to be similar to CNS tissue properties, which is a key requirement for spinal cord repair (Georges et al., 2006). Furthermore, the hydrogel can be developed as injectable formats that can fully plug lesion cavities, thus representing a minimally invasive neurosurgical technique (Cigognini et al., 2011; Kabu et al., 2015). In an exploratory pilot study, we injected the S-220-loaded Fmoc hydrogel into the lesion cavity of rats with contusion SCI at a subacute stage (i.e., 3 weeks after injury) and found significantly improved locomotor functional recovery compared with animals with contusion only. However, further in vivo immunohistochemical and functional studies are required to validate the potential of these treatments in SCI, which is beyond the scope of the current study. Future studies also need to further optimize the injected hydrogel volume and S-220 dose and establish the in vivo effects of S-220 on axonal outgrowth and glial activation.

The failure to find a cure for spinal cord repair is mainly due to the complex injury nature of SCI. Many studies have designed strategies, either alone or in combination, to tackle various obstacles presented by the injury, but so far have failed to translate results to the clinic. Our findings using Epac2 elevation to promote spinal cord repair underpin a significant game change not only in directly enhancing the intrinsic capacity of injured neurons to regrow, but moreover in harnessing the inhibitory environment, including the glial scar and microglia, to facilitate axonal outgrowth. However, caution is needed when extrapolating our strategy to other CNS injuries, such as brain injury and stroke, as recent preclinical evidence suggests that, in the acute phase of hemorrhage-induced brain trauma, increased neuronal expression of Epac2 leads to apoptosis (Zhuang et al., 2019). Future studies are required to fully understand the role of Epac2 under different CNS injury pathogeneses.

\section{References}

Alakpa EV, Jayawarna V, Lampel A, Burgess KV, West CC, Bakker SCJ, Roy S, Javid N, Fleming S, Lamprou DA, Yang JL, Miller A, Urquhart AJ, Frederix PW, Hunt NT, Peault B, Ulijn RV, Dalby MJ (2016) Tunable supramolecular hydrogels for selection of lineage-guiding metabolites in stem cell cultures. Chem 1:298-319.

Anderson MA, Burda JE, Ren Y, Ao Y, O'Shea TM, Kawaguchi R, Coppola G, Khakh BS, Deming TJ, Sofroniew MV (2016) Astrocyte scar formation aids central nervous system axon regeneration. Nature 532:195-200.

Batty NJ, Fenrich KK, Fouad K (2017) The role of cAMP and its downstream targets in neurite growth in the adult nervous system. Neurosci Lett 652:56-63.

Blackmore MG, Wang Z, Lerch JK, Motti D, Zhang YP, Shields CB, Lee JK, Goldberg JL, Lemmon VP, Bixby JL (2012) Kruppel-like factor 7 engineered for transcriptional activation promotes axon regeneration in the adult corticospinal tract. Proc Natl Acad Sci U S A 109:7517-7522.

Boomkamp SD, McGrath MA, Houslay MD, Barnett SC (2014) Epac and the high affinity rolipram binding conformer of PDE4 modulate neurite outgrowth and myelination using an in vitro spinal cord injury model. Br J Pharmacol 171:2385-2398.

Bos JL, de Bruyn K, Enserink J, Kuiperij B, Rangarajan S, Rehmann H, Riedl J, de Rooij J, van Mansfeld F, Zwartkruis F (2003) The role of Rap1 in integrin-mediated cell adhesion. Biochem Soc Trans 31:83-86.

Cafferty WB, Gardiner NJ, Das P, Qiu J, McMahon SB, Thompson SW (2004) Conditioning injury-induced spinal axon regeneration fails in interleukin-6 knock-out mice. J Neurosci 24:4432-4443.

Cheah M, Andrews MR (2018) Integrin activation: implications for axon regeneration. Cells 7:E20.

Cheng X, Ji Z, Tsalkova T, Mei F (2008) Epac and PKA: a tale of two intracellular cAMP receptors. Acta Biochim Biophys Sin (Shanghai) 40:651662. 
Cho JS, Park HW, Park SK, Roh S, Kang SK, Paik KS, Chang MS (2009) Transplantation of mesenchymal stem cells enhances axonal outgrowth and cell survival in an organotypic spinal cord slice culture. Neurosci Lett 454:43-48.

Christensen AE, Selheim F, de Rooij J, Dremier S, Schwede F, Dao KK, Martinez A, Maenhaut C, Bos JL, Genieser HG, Døskeland SO (2003) cAMP analog mapping of Epacl and cAMP kinase. discriminating analogs demonstrate that Epac and cAMP kinase act synergistically to promote PC-12 cell neurite extension. J Biol Chem 278:35394-35402.

Cigognini D, Satta A, Colleoni B, Silva D, Donegà M, Antonini S, Gelain F (2011) Evaluation of early and late effects into the acute spinal cord injury of an injectable functionalized self-assembling scaffold. PLoS One 6:e19782.

Condic ML, Snow DM, Letourneau PC (1999) Embryonic neurons adapt to the inhibitory proteoglycan aggrecan by increasing integrin expression. J Neurosci 19:10036-10043.

Costa LM, Pereira JE, Filipe VM, Magalhães LG, Couto PA, Gonzalo-Orden JM, Raimondo S, Geuna S, Mauricio AC, Nikulina E, Filbin MT, Varejão AS (2013) Rolipram promotes functional recovery after contusive thoracic spinal cord injury in rats. Behav Brain Res 243:66-73.

Cregg JM, DePaul MA, Filous AR, Lang BT, Tran A, Silver J (2014) Functional regeneration beyond the glial scar. Exp Neurol 253:197-207.

Danilov CA, Steward O (2015) Conditional genetic deletion of PTEN after a spinal cord injury enhances regenerative growth of CST axons and motor function recovery in mice. Exp Neurol 266:147-160.

Deng J, Zhang XL, Wang JW, Teng LL, Ge J, Takemori H, Xiong ZQ, Zhou Y (2009) Expression and regulated nuclear transport of transducers of regulated CREB 1 in retinal ganglion cells. Neuroscience 159:1023-1031.

Du K, Zheng S, Zhang Q, Li S, Gao X, Wang J, Jiang L, Liu K (2015) Pten deletion promotes regrowth of corticospinal tract axons 1 year after spinal cord injury. J Neurosci 35:9754-9763.

Dull T, Zufferey R, Kelly M, Mandel RJ, Nguyen M, Trono D, Naldini L (1998) A third-generation lentivirus vector with a conditional packaging system. J Virol 72:8463-8471.

Enserink JM, Christensen AE, de Rooij J, van Triest M, Schwede F, Genieser HG, Døskeland SO, Blank JL, Bos JL (2002) A novel Epac-specific cAMP analogue demonstrates independent regulation of Rap1 and ERK. Nat Cell Biol 4:901-906.

Fawcett JW (2006) Overcoming inhibition in the damaged spinal cord. J Neurotrauma 23:371-383.

Garcia AD, Doan NB, Imura T, Bush TG, Sofroniew MV (2004) GFAPexpressing progenitors are the principal source of constitutive neurogenesis in adult mouse forebrain. Nat Neurosci 7:1233-1241.

Georges PC, Miller WJ, Meaney DF, Sawyer ES, Janmey PA (2006) Matrices with compliance comparable to that of brain tissue select neuronal over glial growth in mixed cortical cultures. Biophys J 90:3012-3018.

Georgieva M (2018) The potential of omega-3 polyunsaturated fatty acids in the prevention and treatment of central neuropathic pain after spinal cord injury. In: Aberdeen, United Kingdom: School of Medicine, Medical Sciences and Nutrition, University of Aberdeen.

Georgieva M, Leeson-Payne A, Dumitrascuta M, Rajnicek A, Malcangio M, Huang W (2018) A refined rat primary neonatal microglial culture method that reduces time, cost and animal use. J Neurosci Methods 304: 92-102.

Ghasemlou N, Bouhy D, Yang J, López-Vales R, Haber M, Thuraisingam T, He G, Radzioch D, Ding A, David S (2010) Beneficial effects of secretory leukocyte protease inhibitor after spinal cord injury. Brain 133:126-138.

Ghosh M, Xu Y, Pearse DD (2016) Cyclic AMP is a key regulator of M1 to $\mathrm{M} 2 \mathrm{a}$ phenotypic conversion of microglia in the presence of Th2 cytokines. J Neuroinflammation 13:9.

Gutilla EA, Steward O (2016) Selective neuronal PTEN deletion: can we take the brakes off of growth without losing control? Neural Regen Res 11: 1201-1203.

Gutilla EA, Buyukozturk MM, Steward O (2016) Long-term consequences of conditional genetic deletion of PTEN in the sensorimotor cortex of neonatal mice. Exp Neurol 279:27-39.

Hannila SS, Filbin MT (2008) The role of cyclic AMP signaling in promoting axonal regeneration after spinal cord injury. Exp Neurol 209:321-332.

Kabu S, Gao Y, Kwon BK, Labhasetwar V (2015) Drug delivery, cell-based therapies, and tissue engineering approaches for spinal cord injury. J Control Release 219:141-154.

Kiermayer S, Biondi RM, Imig J, Plotz G, Haupenthal J, Zeuzem S, Piiper A
(2005) Epac activation converts cAMP from a proliferative into a differentiation signal in PC12 cells. Mol Biol Cell 16:5639-5648.

Kjell J, Olson L (2016) Rat models of spinal cord injury: from pathology to potential therapies. Dis Model Mech 9:1125-1137.

Klarenbeek JB, Goedhart J, Hink MA, Gadella TW, Jalink K (2011) A mTurquoise-based cAMP sensor for both FLIM and ratiometric read-out has improved dynamic range. PLoS One 6:e19170.

Kozlowski C, Weimer RM (2012) An automated method to quantify microglia morphology and application to monitor activation state longitudinally in vivo. PLoS One 7:e31814.

Liu RY, Snider WD (2001) Different signaling pathways mediate regenerative versus developmental sensory axon growth. J Neurosci 21:RC164.

Mei FC, Qiao J, Tsygankova OM, Meinkoth JL, Quilliam LA, Cheng X (2002) Differential signaling of cyclic AMP: opposing effects of exchange protein directly activated by cyclic AMP and cAMP-dependent protein kinase on protein kinase B activation. J Biol Chem 277:11497-11504.

Muñoz-Llancao P, Henríquez DR, Wilson C, Bodaleo F, Boddeke EW, Lezoualc'h F, Schmidt M, González-Billault C (2015) Exchange protein directly activated by cAMP (EPAC) regulates neuronal polarization through Rap1B. J Neurosci 35:11315-11329.

Murphy MM, Zayed MA, Evans A, Parker CE, Ataga KI, Telen MJ, Parise LV (2005) Role of Rap1 in promoting sickle red blood cell adhesion to laminin via BCAM/LU. Blood 105:3322-3329.

Murray AJ, Shewan DA (2008) Epac mediates cyclic AMP-dependent axon growth, guidance and regeneration. Mol Cell Neurosci 38:578-588.

Murray AJ, Tucker SJ, Shewan DA (2009) cAMP-dependent axon guidance is distinctly regulated by Epac and protein kinase A. J Neurosci 29: 15434-15444.

Neumann S, Bradke F, Tessier-Lavigne M, Basbaum AI (2002) Regeneration of sensory axons within the injured spinal cord induced by intraganglionic cAMP elevation. Neuron 34:885-893.

Nijholt IM, Dolga AM, Ostroveanu A, Luiten PG, Schmidt M, Eisel UL (2008) Neuronal AKAP150 coordinates PKA and Epac-mediated PKB/ Akt phosphorylation. Cell Signal 20:1715-1724.

Nikulina E, Tidwell JL, Dai HN, Bregman BS, Filbin MT (2004) The phosphodiesterase inhibitor rolipram delivered after a spinal cord lesion promotes axonal regeneration and functional recovery. Proc Natl Acad Sci U S A 101:8786-8790.

O’Neill P, Lindsay SL, Pantiru A, Guimond SE, Fagoe N, Verhaagen J, Turnbull JE, Riddell JS, Barnett SC (2017) Sulfatase-mediated manipulation of the astrocyte-Schwann cell interface. Glia 65:19-33.

Paco S, Hummel M, Plá V, Sumoy L, Aguado F (2016) Cyclic AMP signaling restricts activation and promotes maturation and antioxidant defenses in astrocytes. BMC Genomics 17:304

Peace AG, Shewan DA (2011) New perspectives in cyclic AMP-mediated axon growth and guidance: the emerging epoch of Epac. Brain Res Bull 84:280-288.

Perale G, Rossi F, Santoro M, Peviani M, Papa S, Llupi D, Torriani P, Micotti E, Previdi S, Cervo L, Sundström E, Boccaccini AR, Masi M, Forloni G, Veglianese P (2012) Multiple drug delivery hydrogel system for spinal cord injury repair strategies. J Control Release 159:271-280.

Qiu J, Cai D, Dai H, McAtee M, Hoffman PN, Bregman BS, Filbin MT (2002) Spinal axon regeneration induced by elevation of cyclic AMP. Neuron 34:895-903.

Qiu W, Zhuang S, von Lintig FC, Boss GR, Pilz RB (2000) Cell type-specific regulation of B-raf kinase by CAMP and 14-3-3 proteins. J Biol Chem 275:31921-31929.

Robichaux WG 3rd, Cheng X (2018) Intracellular cAMP sensor EPAC: physiology, pathophysiology, and therapeutics development. Physiol Rev 98:919-1053.

Schwede F, Bertinetti D, Langerijs CN, Hadders MA, Wienk H, Ellenbroek JH, de Koning EJ, Bos JL, Herberg FW, Genieser HG, Janssen RA, Rehmann H (2015) Structure-guided design of selective Epac1 and Epac2 agonists. PLoS Biol 13:e1002038.

Siddiq MM, Hannila SS (2015) Looking downstream: the role of cyclic AMP-regulated genes in axonal regeneration. Front Mol Neurosci 8:26.

Smith GM, Rutishauser U, Silver J, Miller RH (1990) Maturation of astrocytes in vitro alters the extent and molecular basis of neurite outgrowth. Dev Biol 138:377-390.

Sofroniew MV (2009) Molecular dissection of reactive astrogliosis and glial scar formation. Trends Neurosci 32:638-647.

Steininger TS, Stutz H, Kerschbaum HH (2011) Beta-adrenergic stimula- 
tion suppresses phagocytosis via Epac activation in murine microglial cells. Brain Res 1407:1-12.

Takanaga H, Yoshitake T, Hara S, Yamasaki C, Kunimoto M (2004) cAMPinduced astrocytic differentiation of C6 glioma cells is mediated by autocrine interleukin-6. J Biol Chem 279:15441-15447.

Tan CL, Kwok JC, Patani R, Ffrench-Constant C, Chandran S, Fawcett JW (2011) Integrin activation promotes axon growth on inhibitory chondroitin sulfate proteoglycans by enhancing integrin signaling. J Neurosci 31:6289-6295.

Torres-Espín A, Santos D, González-Pérez F, del Valle J, Navarro X (2014) Neurite-J: an image-J plug-in for axonal growth analysis in organotypic cultures. J Neurosci Methods 236:26-39.

Tucker SJ (2014) The use of fluorescence resonance energy transfer (FRET) to measure axon growth and guidance-related intracellular signalling in live dorsal root ganglia neuronal growth cones. Methods Mol Biol 1162:29-40.

Tukmachev D, Forostyak S, Koci Z, Zaviskova K, Vackova I, Vyborny K, Sandvig I, Sandvig A, Medberry CJ, Badylak SF, Sykova E, Kubinova S (2016) Injectable extracellular matrix hydrogels as scaffolds for spinal cord injury repair. Tissue Eng Part A 22:306-317.

Wang Z, Dillon TJ, Pokala V, Mishra S, Labudda K, Hunter B, Stork PJ (2006) Rap1-mediated activation of extracellular signal-regulated kinases by cyclic AMP is dependent on the mode of Rap1 activation. Mol Cell Biol 26:2130-2145.

Wang Z, Winsor K, Nienhaus C, Hess E, Blackmore MG (2017) Combined chondroitinase and KLF7 expression reduce net retraction of sensory and CST axons from sites of spinal injury. Neurobiol Dis 99:24-35.

Wanner IB, Anderson MA, Song B, Levine J, Fernandez A, Gray-Thompson Z, Ao Y, Sofroniew MV (2013) Glial scar borders are formed by newly proliferated, elongated astrocytes that interact to corral inflammatory and fibrotic cells via STAT3-dependent mechanisms after spinal cord injury. J Neurosci 33:12870-12886.

Wei D, Hurd C, Galleguillos D, Singh J, Fenrich KK, Webber CA, Sipione S, Fouad K (2016) Inhibiting cortical protein kinase A in spinal cord injured rats enhances efficacy of rehabilitative training. Exp Neurol 283:365-374.

Weightman AP, Pickard MR, Yang Y, Chari DM (2014) An in vitro spinal cord injury model to screen neuroregenerative materials. Biomaterials 35:3756-3765.

Wilems TS, Sakiyama-Elbert SE (2015) Sustained dual drug delivery of antiinhibitory molecules for treatment of spinal cord injury. J Control Release 213:103-111.

Young W (2002) Spinal cord contusion models. Prog Brain Res 137:231255.

Zhou H, Li X, Wu Q, Li F, Fu Z, Liu C, Liang Z, Chu T, Wang T, Lu L, Ning G, Kong X, Feng S (2015) shRNA against PTEN promotes neurite outgrowth of cortical neurons and functional recovery in spinal cord contusion rats. Regen Med 10:411-429.

Zhou M, Smith AM, Das AK, Hodson NW, Collins RF, Ulijn RV, Gough JE (2009) Self-assembled peptide-based hydrogels as scaffolds for anchorage-dependent cells. Biomaterials 30:2523-2530.

Zhu J, Marchant RE (2011) Design properties of hydrogel tissueengineering scaffolds. Expert Rev Med Devices 8:607-626.

Zhuang Y, Xu H, Richard SA, Cao J, Li H, Shen H, Yu Z, Zhang J, Wang Z, Li X, Chen G (2019) Inhibition of EPAC2 attenuates intracerebral hemorrhage-induced secondary brain injury via the p38/BIM/Caspase-3 pathway. J Mol Neurosci 67:353-363. 